\title{
The practical Pomeron for high energy proton collimation
}

\author{
R. B. Appleby ${ }^{1, a}$, R. J. Barlow ${ }^{2}$, J. G. Molson ${ }^{3}$, M. Serluca ${ }^{4}$, A. Toader ${ }^{2}$ \\ ${ }^{1}$ The Cockcroft Institute, University of Manchester, Oxford Road, Manchester M13 9PL, UK \\ 2 The University of Huddersfield, Huddersfield HD1 3DH, UK \\ ${ }^{3}$ LAL, Univ. Paris-Sud, CNRS/IN2P3, Université Paris-Saclay, Orsay, France \\ ${ }^{4}$ CERN, 1203 Geneva, Switzerland
}

Received: 26 April 2016 / Accepted: 11 September 2016 / Published online: 26 September 2016

(c) The Author(s) 2016. This article is published with open access at Springerlink.com

\begin{abstract}
We present a model which describes proton scattering data from ISR to Tevatron energies, and which can be applied to collimation in high energy accelerators, such as the LHC and FCC. Collimators remove beam halo particles, so that they do not impinge on vulnerable regions of the machine, such as the superconducting magnets and the experimental areas. In simulating the effect of the collimator jaws it is crucial to model the scattering of protons at small momentum transfer $t$, as these protons can subsequently survive several turns of the ring before being lost. At high energies these soft processes are well described by Pomeron exchange models. We study the behaviour of elastic and single-diffractive dissociation cross sections over a wide range of energy, and show that the model can be used as a global description of the wide variety of high energy elastic and diffractive data presently available. In particular it models low mass diffraction dissociation, where a rich resonance structure is present, and thus predicts the differential and integrated cross sections in the kinematical range appropriate to the LHC. We incorporate the physics of this model into the beam tracking code MERLIN and use it to simulate the resulting loss maps of the beam halo lost in the collimators in the LHC.
\end{abstract}

\section{Introduction and motivation}

The world's highest energy particle accelerator, the Large Hadron Collider (LHC), contains two high-energy proton beams travelling in opposite directions, guided around the accelerator ring by superconducting (SC) magnets. Its nominal stored beam energy of $360 \mathrm{MJ}$ is orders of magnitude greater than previous accelerators, such as the Tevatron. This high energy stored beam passes in the machine aperture close to its magnet SC coils with a quench limit of about

\footnotetext{
a e-mail: robert.appleby@manchester.ac.uk
}

$40 \mathrm{~mW} / \mathrm{cm}^{3}$ at the operational current of $80 \%$ of the magnet critical current [1]. The limit corresponds to the design limit of $13-15 \mathrm{~mW} / \mathrm{cm}^{3}$ including an assumed safety factor of 3 [2]. A powerful cleaning system is vital to the machine protection in order to operate below the quench limit, with a highly efficient collimation system necessary in order to remove any stray halo protons. The halo is generated by various effects [2] and it is characterised as an off-momentum halo (in which particle energies deviate from the reference) and a betatron halo (in which particles have large transverse amplitudes). Although the collimation system is adequate for the current configuration of the LHC, for the future HighLuminosity (Hi-Lumi) machine [3] upgrade the physics of the scattering of protons in the collimators must be accurately simulated, to avoid any quench of the SC magnets and to protect the vulnerable parts of the machine such as the detectors.

The tracking of protons around the ring and inside the collimator material is based on complex simulations where many different physics effects are involved. Here we focus on the scattering. Protons interact with both electrons and nuclei in the collimator material, with the former giving ionisation energy loss. The latter can be divided into elastic $(p p \rightarrow p p)$, Single-Diffractive (SD) ( $p p \rightarrow p X$ or $p p \rightarrow X p)$, double diffractive $(p p \rightarrow X Y)$ and inelastic scatters. Note that we ignore nuclear effects and consider a nucleus as a collection of protons and neutrons, and interactions with neutrons are treated similarly to those with protons. Experimentally $p p$ and $p n$ cross sections are within less than $2 \%$ at the highestenergy $p n$ data available, $\sqrt{s}=30 \mathrm{GeV}$, and theoretically the agreement is expected to be even less than this, as the relevant processes are dominated by Pomeron and $f_{2}$ exchange. This approximation has also been used in previous studies of the LHC collimation system ([4,5] and references therein), which agree with measured losses.

To study the beam halo we do not consider inelastic scatters, double-diffractive scatters, or SD interactions $p p \rightarrow$ 
$X p$, in which the beam proton breaks up: for such events all the energy is lost locally, within $50 \mathrm{~m}$, according to studies by the CERN collimator group [6,7]. With elastic and singlediffractive scattering $(p p \rightarrow p X)$ the emerging protons are only slightly affected and may survive several turns before being lost. The elastic scattering contributes to the betatron halo creation, and SD to the off-momentum halo.

The LHC ring is divided into 8 regions. For the nominal layout, as described in the design report [2], there are two collimation regions. In the third Interaction Region (IR3), the removal of off-momentum halo particles, known as momentum cleaning, takes place in a dispersive region. In IR7, particles with large transverse amplitude are removed; this is known as betatron cleaning. There is also an accelerating region in IR4, and a beam dump region in IR6. The remaining four regions are dedicated to the detector insertions: there are two at low $\beta^{*}$ in IR1 (ATLAS) and IR5 (CMS), and two at high $\beta^{*}$ in IR2 (ALICE) and IR8 (LHCb), where $\beta^{*}$ is the betatron function of the magnetic lattice at the interaction point. In each collimation region there is a cleaning hierarchy, and the primary collimators (TCP) in IR7 have the tightest apertures of the machine. In addition, tertiary collimators (TCT) are installed at both sides of the detector insertions to protect the final focus SC magnets and detectors.

In Table 1, the equivalent centre-of-momentum energy is given for various LHC proton energies on a 'fixed target' proton in the collimator. It varies from $29 \mathrm{GeV}$ at injection $\left(E_{\text {beam }}=450 \mathrm{GeV}\right)$ to $115 \mathrm{GeV}$ for the nominal beam energy, $176 \mathrm{GeV}$ for the LHC energy upgrade and $306 \mathrm{GeV}$ for the FCC-hh.

Experimental data for $p p$ and $p \bar{p}$ reactions exist for many energies from different experiments and accelerators, principally the Intersecting Storage Rings (ISR) at $\sqrt{s}=23-$ $63 \mathrm{GeV}$ and the Tevatron at $2 \mathrm{TeV}$. There are also data from the SPPיS. With plentiful data both above and below the range required, our model parameters are obtained by interpolation, rather than extrapolation.

In this paper we create a model within the Pomeron and Reggeon exchange framework of Donnachie and Landshoff $[8,9]$. The model is an elegant description of the strong interaction at high energies, and describes the experimental

Table 1 The relevant beam energies required for protons impinging on a collimator

\begin{tabular}{lcc}
\hline State & $E_{\text {beam }}[\mathrm{GeV}]$ & $\begin{array}{c}\text { Fixed target } \\
\sqrt{s}[\mathrm{GeV}]\end{array}$ \\
\hline LHC injection & 450 & 29 \\
LHC 2011 collision & 3500 & 81 \\
LHC 2012 collision & 4000 & 84 \\
LHC nominal collision & 7000 & 115 \\
FCC-hh & 50000 & 306 \\
\hline
\end{tabular}

data for total, elastic and SD scattering with minimal assumptions. The fit uses a small number of parameters to describe data for 21 energies and 11 experiments, aiming to achieve the best possible fit. We use an extension of the model which we fit to most of the available elastic and SD data, in order to obtain a parametrisation which covers the required protontarget kinematical range at LHC energies.

The extended model, which we simply call the DL model, is implemented into the beam tracking library MERLIN [1013], which is then used to simulate the loss maps for the nominal LHC.

We use this model to simulate the LHC loss maps, demonstrating the cleaning performance of the collimation system. This performance determines whether the accelerator can safely run at higher intensity, or whether additional shielding or collimators will be required. Realistic simulations of particle loss maps are fundamental to our ability to predict eventual quenching locations, for the nominal LHC and possible upgraded collimation systems, new materials and advanced collimation concepts such as hollow electron lenses [14] and crystal collimation [15].

The layout of this paper is as follows. In Sect. 2 we introduce the kinematics and discuss the requirements for the simulation of proton scattering within the collimator materials. In Sect. 3 we model the elastic scattering, performing a fit which achieves a good description of the available data. Then in Sect. 4 we describe the single diffractive model and obtain a fit for the double differential cross section for low and high missing mass regions, producing a good description of a wide range of data. We illustrate the fitting procedure and present the results at LHC energies and the prediction of the total $\mathrm{SD}$ cross section as a function of the centre-of-mass energy $\sqrt{s}$. We show that it is possible to use the DL fit approach for elastic and SD scattering to cover the required range of kinematical variables for the LHC. In Sect. 5 we introduce the MERLIN code and the implementation of the model. The resulting loss maps for the nominal $\mathrm{LHC}$ at $7 \mathrm{TeV}$ are presented with a detailed examination of the betatron cleaning region and the losses in the dispersion regions.

The data sources for elastic and single diffraction dissociation at different energies are reported in 1 and 1 , along with references and the fit of the model to data.

\section{Proton scattering and beam dynamics}

\subsection{Particle beam dynamics and dispersion}

The horizontal transverse motion of a particle in an accelerator is given by the Courant-Snyder parameterization of the solution to Hill's equation [16] 


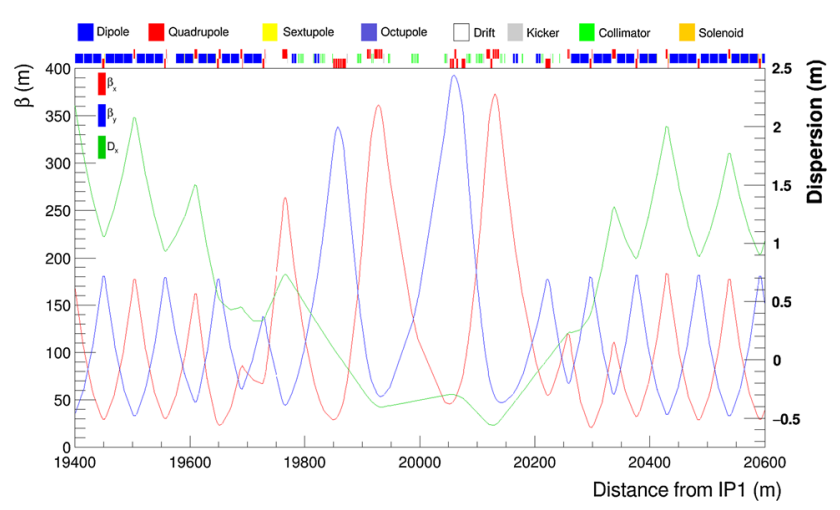

Fig. 1 Horizontal dispersion (green line) and horizontal and vertical $\beta$ functions (red and blue lines) for beam 1 around the IR7 region in the LHC as generated by MERLIN using the V6.503 optics layout. The horizontal axis represents the distance from ATLAS interaction point

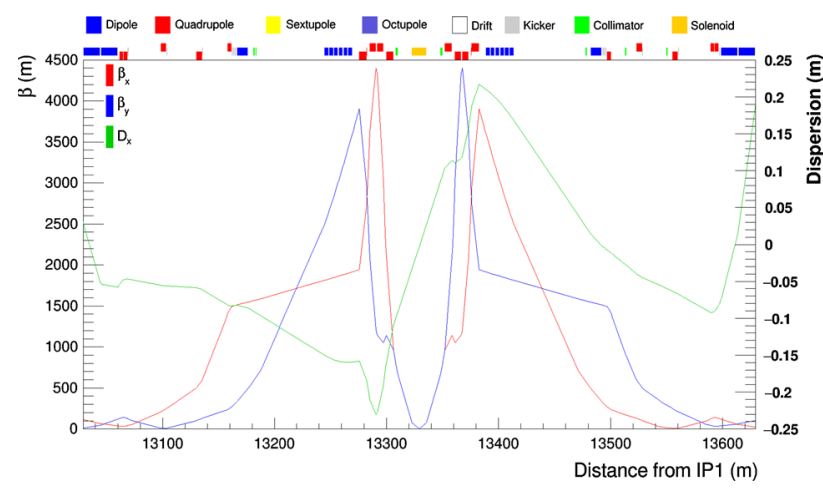

Fig. 2 Horizontal dispersion (green line) and $\beta$ functions (red and blue lines) for beam 1 around the CMS detector (IR5) in the LHC as generated by MERLIN using the V6.503 optics layout

$x\left(s^{\prime}\right)=\sqrt{2 J \beta_{x}\left(s^{\prime}\right)} \sin \left(\mu_{x}\left(s^{\prime}\right)+\mu_{x 0}\right)+D_{x} \frac{\Delta p}{p}$.

Here $s^{\prime}$ is the longitudinal position along the accelerator lattice, $J$ is the particle action, $\beta_{x}$ the betatron function of the accelerator magnetic lattice, and $\mu_{x}$ the betatron phase. $p$ is the reference momentum for the lattice and $\Delta p$ is the deviation of the particle from this reference momentum. $D_{x}$ is the dispersion function, describing the motion of particles with such a deviation. Protons that have lost momentum in diffractive interactions may have large transverse displacements from the reference orbit in regions where $\left|D_{x}\right|$ is large.

Figure 1 shows the $\beta$-functions and the horizontal dispersion in the betatron collimation region IR7. There, the dispersion is small but the $\beta$-functions are large, so the collimators placed here remove protons in the betatron halo but not the energy halo.

Figure 2 shows the $\beta$-functions and horizontal dispersion in the IR5 region, where the CMS detector is located. The magnetic elements are shown above the plot, including the quadrupole triplets on both sides of the detector that squeeze the beam at the interaction point. For the nominal LHC, the value of $\beta$ at the interaction point is $55 \mathrm{~cm}$ in IR1 (ATLAS) and IR5 (CMS) and $10 \mathrm{~m}$ in IR2 (ALICE) and IR8 (LHCb).

\subsection{Kinematics and the relevant range of $t$ and $M_{X}$}

If a proton with mass $M_{p}$ and 4-momentum $p_{i}^{\mu}=\left(E_{i}, \mathbf{p}_{\mathbf{i}}\right)$ in the lab frame interacts with a stationary proton $P^{\mu}=$ $\left(M_{p}, 0\right)$, and scatters to $p_{f}^{\mu}=\left(E_{f}, \mathbf{p}_{\mathbf{f}}\right)$, the invariants $s$ and $t$ are defined as

$s=\left(P^{\mu}+p_{i}^{\mu}\right)^{2}=2 M_{p}^{2}+2 M_{p} E_{i}$,

$t=\left(p_{i}^{\mu}-p_{f}^{\mu}\right)^{2}=\left(E_{i}-E_{f}\right)^{2}-\left(\mathbf{p}_{i}-\mathbf{p}_{f}\right)^{2}$.

$\sqrt{s}$ is the energy in the centre of momentum system. The expression for $t$ can usefully be rewritten in terms of the proton scattering angle $\theta$,

$t=2 M_{p}^{2}-2 E_{i} E_{f}+2 p_{i} p_{f} \cos \theta$.

The invariant mass of the diffracted proton-target, $M_{X}$ also called the missing mass, is given by energy-momentum conservation

$M_{X}^{2}=\left(p_{i}^{\mu}+P^{\mu}-p_{f}^{\mu}\right)^{2}=\left(E_{i}-E_{f}+M_{P}\right)^{2}-\left(\mathbf{p}_{i}-\mathbf{p}_{f}\right)^{2}$.

It is convenient to define the dimensionless variable

$\xi=\frac{M_{X}^{2}}{s}$.

The energy loss by the scattered proton is given by combining Eqs. 4 and 5

$\Delta E=E_{f}-E_{i}=\frac{t+M_{p}^{2}-M_{X}^{2}}{2 M_{p}}$.

(For elastic scattering $M_{X}$ is equal to $M_{p}$ and this simplifies further.) Thus the quantities relevant for the simulation: $\Delta E$ the energy change, and $\theta$, the direction of the outgoing particle (apart from a random azimuthal angle) are determined by the quantities $t$ and $M_{X}$ or $\xi$, and it is the distributions for these two quantities that are predicted by the model.

If the scattering angle is significantly larger than the beam divergence, the scattered proton will be lost immediately, or in the nearby downstream region of the machine. Thus our model of elastic scattering must be accurate at small $|t| /$ small $\theta$ but need not model large $|t| /$ large $\theta$, where 'large' and 'small' refer to comparison of the scattering angle with the angular beam divergence at that location. Table 2 shows the LHC V6.503 machine optics, characterised by the Twiss parameters $\alpha$ and $\beta$, at three typical collimators in IR7 (defined earlier) and, assuming a normalised nominal beam emittance of $3.75 \mathrm{~mm}$.mrad, shows the angular 
Table 2 A list of collimators in IR7 with their Twiss parameters, and assuming a normalised emittance of $3.75 \mu \mathrm{m} \mathrm{rad}$, the value of $|t|$ values corresponding to 20 times the nominal beam divergence at collimator location

\begin{tabular}{lcrllllll}
\hline Collimator & $\beta_{x}$ & $\beta_{y}$ & $\alpha_{x}$ & $\alpha_{y}$ & $\begin{array}{l}\sigma_{\max }^{\prime}(\mu \mathrm{rad}) \\
\text { at 7 TeV }\end{array}$ & $\begin{array}{l}\sigma_{\max }^{\prime}(\mu \mathrm{rad}) \\
\text { at 3.5 TeV }\end{array}$ & $\begin{array}{l}|t|_{\max } \\
\mathrm{TeV}\end{array}$ & $\begin{array}{l}|t|_{\max } \\
3.5 \mathrm{TeV}\end{array}$ \\
\hline D6L7.B1 & 158 & 78 & 2.1 & -1.1 & 4.2 & 5.9 & 0.17 & 0.34 \\
C6L7.B1 & 150 & 83 & 2.0 & -1.2 & 4.2 & 5.9 & 0.17 & 0.34 \\
A6L7.B1 & 129 & 97 & 1.9 & -1.3 & 4.2 & 5.9 & 0.17 & 0.34 \\
EXTREME & 100 & 100 & 3 & 3 & 7.1 & 10.0 & 0.49 & 0.98 \\
\hline
\end{tabular}

divergences corresponding to 20 times their nominal values at the collimator locations, and the corresponding $|t|$ values.

The table shows that, for the collimation optics in IR7, modelling elastic scattering events up to $|t|=0.34 \mathrm{GeV}^{2}$ is sufficient to correctly model scattering events which could change the collimator-induced loss map beyond the immediately vicinity of the primary collimator. The table also includes an extreme case of collimation optics (arbitrarily chosen) which shows that for larger values of $\alpha$ at collimator locations we need to model an approximately double $|t|$ range of elastic events. The elastic fits in this paper are valid over the range of available data, and extend to $t=-14.2 \mathrm{GeV}^{2}$, which is more than ample for our simulations.

Detailed modelling of scattering at very small $|t|$ is also unnecessary as very small angle scatters do not lead to beam loss. To investigate this we have used MERLIN to perform a full phase space aperture scan in both planes, injecting a beam filling one plane of phase space, i.e. a grid in $x$ and $x^{\prime}$, with the other coordinates matched to the optical lattice at each collimator. These particles were then tracked for 100 turns, with particles removed if they touch any aperture restrictions, and the surviving particles' initial angles at the collimator jaws recorded. The smallest possible angle for which a particle is lost gives the minimum $t$ value required. For the collimator jaw around the experimental regions, which have the minimum aperture available for scattering, an appropriate minimum value of $|t|$ is $0.0001 \mathrm{GeV}^{2}$.

The ranges of $M_{X}$ and $|t|$ required for modelling diffractive scattering depend on the beam's angular divergence and its intrinsic energy spread. For the former, referring to Table 2, we take a conservative value of $\sigma^{\prime}=15 \mu \mathrm{rad}$ to cover all possible current and future cases including deviation from the specified normalised emittance value. For the latter, the LHC beam energy spread $\sigma_{e}$ is the nominal $1.1 \cdot 10^{-4}$ at $7 \mathrm{TeV}$, and has been measured to be $1.36 \pm 0.04 \cdot 10^{-4}$ at 3.5 $\mathrm{TeV}$. The dependence of the scattering angle on $\xi$ is weak, and a $|t|$ limit of $4 \mathrm{GeV}^{2}$ corresponds to $20 \sigma^{\prime}$ over all relevant $\xi$. For a $3.5 \mathrm{TeV}$ beam energy, $\xi=0.12$ corresponds to $M_{X}=28 \mathrm{GeV}$ and, even at our maximum $|t|$ of $4 \mathrm{GeV}^{2}$, this gives an energy deviation $420 \mathrm{GeV}$, which is $856 \sigma_{e}$, and also $41 \sigma^{\prime}$. At $7 \mathrm{TeV}$ it corresponds to $1109 \sigma_{e}$, The conclusion for all energies is that a kinematical range of $\xi$ up to 0.12 and $|t|$ up to $4 \mathrm{GeV}^{2}$ is sufficient and conservative for the single diffractive fit. The fits are not sensitive to the minimum values, and we take the fits down to threshold for $\xi$ and down to $t=-0.0001 \mathrm{GeV}^{2}$.

\section{Elastic proton scattering and the Pomeron}

The differential cross-section $d \sigma / d t$ of elastic $p p$ and $p \bar{p}$ scattering is described by Coulomb scattering at very small $|t|$ and nuclear scattering for larger $|t|$. Early measurements at the ISR $[17,18]$ with energies between $23 \mathrm{GeV}$ and $63 \mathrm{GeV}$ revealed at low $|t|$ an approximately exponential behaviour, $e^{-B|t|}$, where $B$ is known as the slope parameter. This is followed by a diffractive minimum at around $|t| \simeq 1.4 \mathrm{GeV}^{2}$, and subsequently a broad peak. The energy dependence of $d \sigma / d t$ shows a shrinkage of the elastic peak, i.e. an increase in $B$, with increasing $\sqrt{s}$ [19].

The DL model includes Regge ( $\rho, \omega$ and $a_{2}, f_{2}$ trajectories) and Pomeron exchange [20], including multiple Regge and Pomeron exchanges [8,21]. At large $t$ triple gluon exchange is also present [22,23]. Recently, in the light of the LHC data from the TOTEM experiment, a hard Pomeron term has also been added [24].

We extend the DL nuclear model to take into account the low $t$ Coulomb peak in order to simulate elastic scattering in the energy ranges given in Table 1 . The DL model has been fitted to all elastic data to obtain the fit parameters of the model. The fitting procedure is different from the one originally used in [24] where the normalisations were kept constant. The details of our approach are given here; a full account can be found in [25].

In this section we describe the elastic model general formulation and the fitting procedures. We then present the fitted differential cross section and the total elastic cross section.

\subsection{The general formulation}

The method used to calculate the elastic differential cross section is well established [26]. It is given by

$\frac{d \sigma}{d t}=\pi\left|f_{c}+f_{n}\right|^{2}$ 
where $f_{c}$ and $f_{n}$ are the Coulomb and nuclear amplitudes, and the formalism includes the interference term. In general, there is a phase difference between the Coulomb and nuclear amplitudes, $e^{i \alpha \phi(t)}$, such that

$\frac{d \sigma}{d t}=\pi\left|f_{c} e^{i \alpha \phi(t)}+f_{n}\right|^{2}$.

To find the Coulomb phase $\phi$, we use a fit to the cross section slope at $t=0$, and the calculation by Cahn [27],

$\phi=\mp\left(\gamma+\ln \left[\frac{B}{2}\right]\right)$.

The upper sign refers to $p p$ scattering, the lower to $p \bar{p}, \gamma$ is Euler's constant and $B$ is given by

$B=8.1+1.2 \log \sqrt{s}$.

\subsubsection{Photon exchange}

The Coulomb amplitude $f_{c}$ is given by [28]

$f_{c}=\mp 2 \alpha_{e m} \frac{F(t)^{2}}{t}$,

where $F(t)$ is the proton electromagnetic form factor, given by equation 3.17 in [9],

$F(t)=\frac{4 M_{p}^{2}-2.79 t}{4 M_{p}^{2}-t} \frac{1}{(1-t / 0.71)^{2}}$.

However it is well approximated by the simpler formula [9],

$F(t)^{2}=0.27 e^{8.38 t}+0.56 e^{3.78 t}+0.18 e^{1.36 t}$.

\subsubsection{Hadronic exchange}

In the DL model, 4 Regge trajectories are used, the hard Pomeron, the soft Pomeron, the $f_{2}$ and $a_{2}$ trajectory, and the $\omega$ and $\rho$ trajectory. In the following these are labeled by 0 to 3 respectively. This gives, for the nuclear amplitude,

$f_{n}=A_{g g g}(s, t)+\sum_{i=0}^{3} A_{i}(s, t)$.

where we have included a triple-gluon exchange amplitude. Its form varies like $1 / t^{4}$ at large $t$, is exponential at small $t$ and the expressions are forced to match at an intermediate $t=t_{0}$, such that [20]

$$
\begin{aligned}
A_{g g g}(t) & =\frac{\sqrt{16 \pi} \sqrt{0.09}}{t^{4}}\left(|t|>\left|t_{0}\right|\right) \\
& =\frac{\sqrt{16 \pi} \sqrt{0.09}}{t_{0}^{4}} \exp \left(4-4 t / t_{0}\right)\left(|t|<\left|t_{0}\right|\right)
\end{aligned}
$$

The amplitude is purely real and energy independent. $t_{0}$ is used as a free parameter in the fitting procedure.

The exchange amplitudes $A_{i}$ are

$$
A_{i}(s, t)=Y_{i}\left(2 v \alpha_{i}^{\prime}\right)^{\alpha_{i}(t)} e^{\frac{i \pi}{2} \alpha_{i}(t)} F^{2}(t),
$$

with

$2 v=\left(\frac{s-u}{2}\right) \quad Y_{i}=-X_{i}(i=0,1,2) \quad Y_{3}=i X_{3}$

where $s$ and $u$ are the Mandelstam variables and $F(t)$ is the form factor of the proton. The $X_{i}$ are real and positive; the factor $i$ multiplying $X_{3}$ ensures the correct signature factor [8] for negative $C$-parity exchange. The amplitude for $p \bar{p}$ scattering is the same except that $Y_{3}$ has the opposite sign.

The form of each Regge trajectory is

$\alpha_{i}(t)=1+\epsilon_{i}+\alpha_{i}^{\prime} t$

where $\alpha_{i}^{\prime}$ is the slope, and $1+\epsilon_{i}$ is the intercept at $t=0$.

We extend this single scattering model to include double Pomeron exchange [24,25], which is necessary to account for the observed dip in the elastic differential cross section. The appropriate term ${ }^{1}$ is included in our computed amplitude; it involves no new parameters apart from parameterising higher order scattering terms not included through scaling the double scattering amplitude by a factor $\lambda$.

\subsubsection{The full model}

Combining the contributions to Eq. (9) gives the differential cross section for elastic scattering as

$$
\begin{aligned}
\frac{d \sigma}{d t}= & \pi\left[A_{c}(s, t)\right]^{2}+\frac{1}{4 \pi}\left(\mathbb{R} e\left[A_{n}(s, t)\right]^{2}+\mathbb{I} m\left[A_{n}(s, t)\right]^{2}\right) \\
& +\left(\rho+\alpha_{e m} \phi\right) A_{c}(s, t) \mathbb{I} m\left[A_{n}(s, t)\right],
\end{aligned}
$$

where the optical theorem has been used for the cross term and we define the ratio of the real and imaginary components of the nuclear term as $\rho$. The real and imaginary terms are the sum of all the corresponding terms from Regge exchange, and both single and double Pomeron exchange. We have assumed that $\alpha \phi$ is small, so that the exponential term could be expanded. For $p \bar{p}$ scattering, the sign of the terms must be inverted for the $C=-1 \omega$ and $\rho$ trajectory, and the triple gluon term.

\subsection{The elastic model fit}

Using this model, we fit all suitable available elastic data. Since the electromagnetic cross section diverges as $t \longrightarrow 0$,

\footnotetext{
1 The full set of equations can be obtained from the authors.
} 
Table 3 The fitted parameters for the elastic scattering model

\begin{tabular}{lll}
\hline Parameter & Value & Fit uncertainty \\
\hline$X_{0}$ & 228 & 12 \\
$X_{1}$ & 194 & 2 \\
$X_{2}$ & 519 & 24 \\
$X_{3}$ & 10.8 & 3.3 \\
$\epsilon_{0}$ & 0.1062 & 0.0007 \\
$\epsilon_{1}$ & 0.0972 & 0.0002 \\
$\epsilon_{2}$ & -0.511 & 0.007 \\
$\epsilon_{3}$ & -0.3 & 0.05 \\
$\alpha_{0}^{\prime}$ & 0.045 & 0.003 \\
$\alpha_{1}^{\prime}$ & 0.28 & 0.001 \\
$\alpha_{2}^{\prime}$ & 0.82 & Fixed \\
$\alpha_{3}^{\prime}$ & 0.90 & Fixed \\
$\lambda$ & 0.5212 & 0.0006 \\
$t_{0}$ & 5.03 & 0.01 \\
\hline
\end{tabular}

a minimum $t$ value must be defined otherwise the integrated cross section will be infinite.

Using MINUIT within ROOT [29], a global fit is performed over the data shown in Table 11 of 1, where $\sqrt{s}>23 \mathrm{GeV}$. At and below this value, the fit quality of the model starts to degrade.

In the fitting, both the Regge trajectories in the model are "effective" trajectories and are initialized with values taken from a Chew-Frautschi plot. The Pomeron trajectories, $X_{i}$ factors, and both $\lambda$ and $t_{0}$ are taken as free parameters, making a total of 14 parameters. However the Regge trajectory slopes are fixed to control the stability of the fit. Full systematic uncertainties are taken into account and correlations between experimental data sets are included. The fit is performed over all data, and over the full $t$ range available, yielding a $\chi^{2} / N D F=4.00$. This overall figure covers a considerable variation: for many datasets the fit quality is acceptable $\left(\chi^{2} / N D F \sim 1\right)$ but there are some features of some datasets where the model and the data systematically disagree in terms of the statistical errors, which are, particularly at low $t$ in the peak, sometimes very small.

The resulting fit parameters and uncertainties given by MINUIT are given in Table 3. We note the two Pomeron intercepts are both soft $\left(\epsilon_{0}\right.$ and $\left.\epsilon_{1}\right)$-in essence we started off with a hard and a soft Pomeron and the fit to the data pushed them together, leaving a single soft Pomeron $[30,31]$ with a complicated $t$ dependence.

\subsection{Total and differential elastic cross section}

The total elastic cross section is usually quoted as the contribution from the nuclear term only,
Table 4 The integrated elastic proton-proton cross section obtained from integration over the differential cross section at LHC energies

\begin{tabular}{lcl}
\hline Energy $[\mathrm{GeV}]$ & $\sqrt{s}[\mathrm{GeV}]$ & $\sigma_{e l}[\mathrm{mb}]$ \\
\hline 450 & 29.0 & 6.8 \\
3500 & 81.0 & 8.1 \\
4000 & 83.9 & 8.2 \\
7000 & 114.6 & 8.8 \\
\hline
\end{tabular}

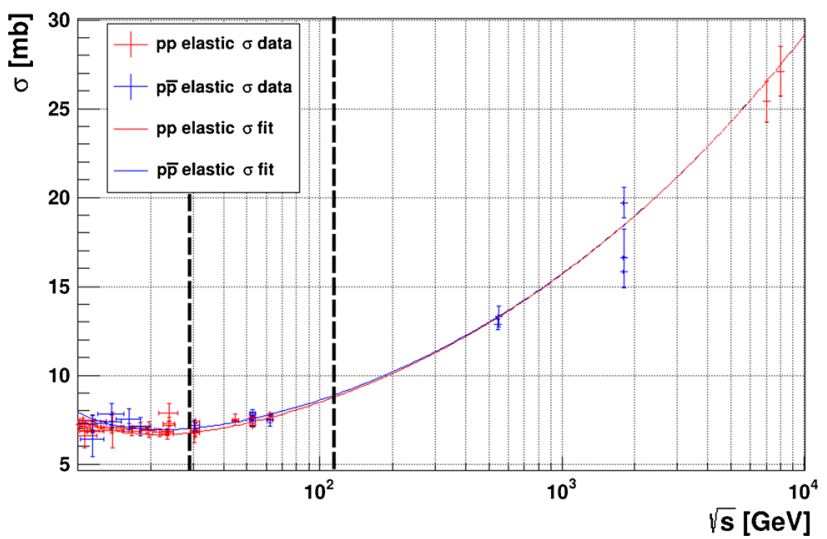

Fig. 3 The total elastic cross section fit against experimental $p p$ and $p \bar{p}$ data. The black dashed vertical lines at 29 and $114.6 \mathrm{GeV}(\sqrt{s})$, that is a beam energy of 450 and $7000 \mathrm{GeV}$, show the energy range of interest for the LHC collimation system

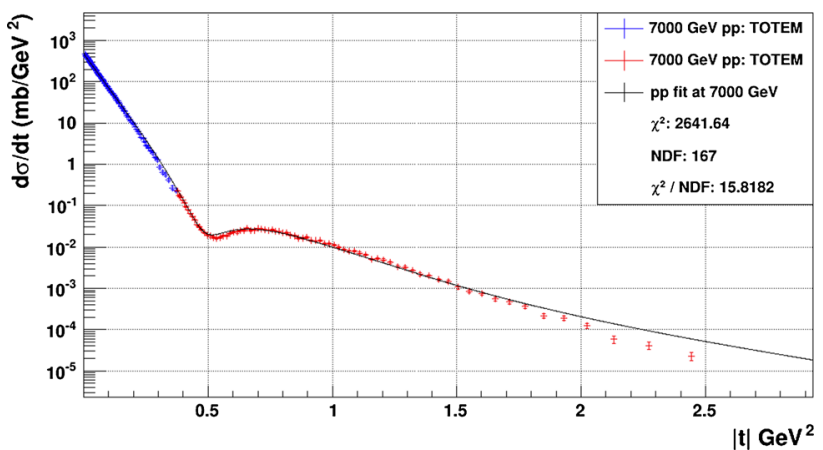

Fig. 4 The $p p$ elastic scattering model model fit (fitted over all data) shown for $\sqrt{s}=7000 \mathrm{GeV}$, over the full published $t$ range

$\sigma_{e l}=\pi \int\left|f_{n}(t)\right|^{2} d t$

Our values, calculated by integration over the differential cross section according to this convention, for the total elastic $p p$ cross sections at LHC energies are listed in Table 4.

In Fig. 3 we show a subset of results for the total elastic cross section fit against experimental $p p$ and $p \bar{p}$ data. The black dashed vertical lines at 450 and $7000 \mathrm{GeV}$ show the energy range of interest for the LHC collimation system.

Figure 4 shows the fit for $p p$ elastic scattering at $\sqrt{s}=$ $7000 \mathrm{GeV}$ using TOTEM data from the LHC, showing the fit performance at very high energy. Figure 5 shows the fit 


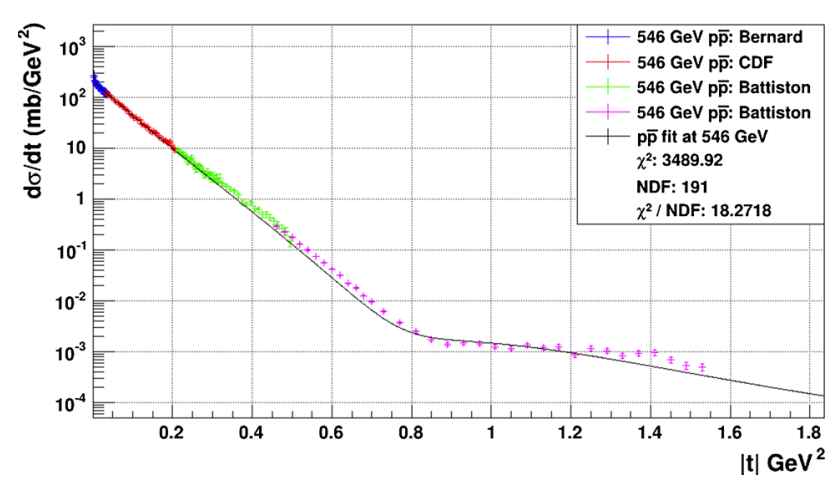

Fig. 5 The $p \bar{p}$ elastic scattering model model fit (fitted over all data) shown for $\sqrt{s}=546 \mathrm{GeV}$, over the full published $t$ range

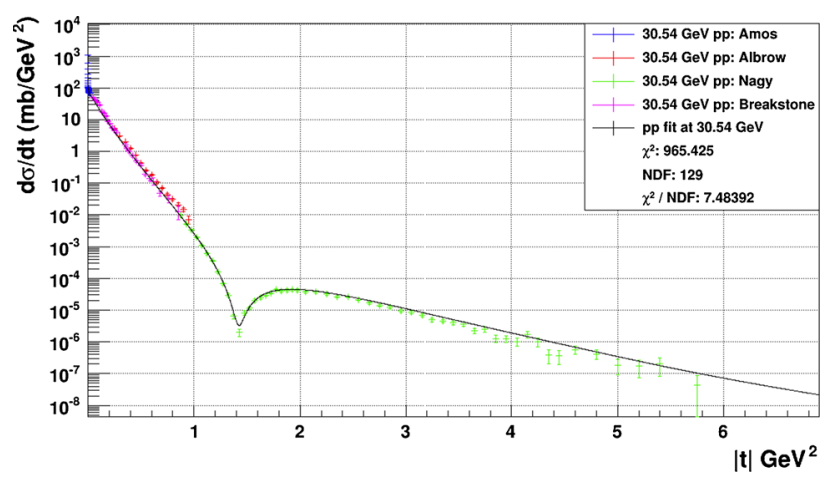

Fig. 6 The $p p$ elastic scattering model model fit (fitted over all data) shown for $\sqrt{s}=30.54 \mathrm{GeV}$, over the full published $t$ range

for $p \bar{p}$ elastic scattering from $\sqrt{s}=546 \mathrm{GeV}$, showing the fit performance in an energy range just above the range for proton interactions in collimators. Finally, Fig. 6 shows the fit for $p p$ elastic scattering from $\sqrt{s}=30.54 \mathrm{GeV}$, showing the accurate description of the elastic dip for this kinematic region.

The remaining plots for $p p$ and $p \bar{p}$ elastic scattering are presented in 1.

All figures show the combined systematic and statistical errors. Each plot shows the differential $d \sigma / d t$ distribution, with each experimental data set in a different colour. The black line is the fitted function with the parameters given in
Table 3. The normalisations given in 1 have been applied to the data.

\section{Single diffraction dissociation}

Single diffraction dissociation in $p p$ interactions is the process

$p p \rightarrow p X$,

in which one proton breaks up into a system $X$ while the other scatters elastically. Diffractive kinematics are described by $s, t$ and $M_{X}$. In fixed-target SD events at LHC energies $M_{X}$ can vary from $M_{p}+m_{\pi}$ to more than $50 \mathrm{GeV}$.

The simplest description of high energy process is given in the diagram of Fig. 7a in which a Reggeon or a Pomeron is exchanged between the elastically-scattered proton and the system $X$. In the limit $s \gg M_{X}^{2} \gg|t|$ and $M_{X}^{2}$ not too small the process may be described by the triple-Regge model $[8,9,32-34]$ as illustrated in Fig. $7 \mathrm{c}$ and discussed in Sect. 4.1. For small values of the missing mass $M_{X}$, around a few $\mathrm{GeV}$, the system $X$ is dominated by baryon resonances and requires a different treatment. A simple model [35], based on duality arguments, allows us to extend the fit to low mass where existing data are scarce. This is discussed in Sect. 4.2.

The advent of the LHC has renewed interest in diffraction dissociation [36-39]. The associated models go beyond the simple triple-Regge model, principally by the inclusion of absorptive corrections, and they are successful in describing the total single diffraction cross section and, in some cases, the double differential cross section $d^{2} \sigma / d t d \xi$ at small $t$. In one sense our approach is less ambitious in that we use the standard triple-Regge model without modification. In another sense, however, it is much more ambitious as we attempt, successfully, to describe all existing single diffractive dissociation data in $p p$ interactions.

\subsection{High mass: triple-Regge formalism}

The triple-Regge description, shown in Fig. 7, describes the $p p$ SD cross section in the region of high $\xi$ as the sum of

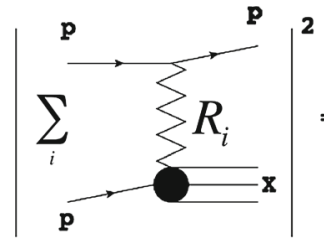

(a)

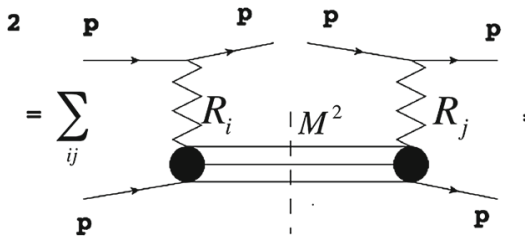

(b)

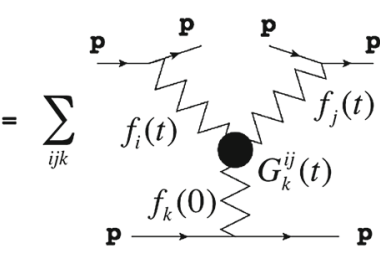

(c)
Fig. 7 The triple-Regge description of high-mass diffractive dissociation. a The squared amplitude summed over all possible system $X$ in the large triple-Regge limit $\left(s \gg M_{X}^{2} \gg|t|\right)$. b The discontinuity across
$M_{X}^{2}$ of the scattering amplitude. $\mathbf{c}$ The total cross section $d^{2} \sigma / d t d \xi$ as the sum of triple-Reggeon contributions 
contributions from triple-Regge exchanges [8] (and applied to the LHC in [34]). In Fig. 7c each of the upper exchanges carry momentum transfer $t$ while the lower one carries zero momentum transfer; the $f_{i}(t), i=1,2,3$ are the couplings of the exchanges to the relevant hadrons and $G_{3}^{12}(t)$ is the triple-Reggeon vertex. In addition to the Pomeron, Reggeised $f_{2}, a_{2}, \omega, \rho$ exchanges are allowed so in principle we require a whole series of terms, given by

$\begin{array}{lllllllll}\mathbb{P P} & \mathbb{P P} & \mathbb{R} R & \mathbb{R} R & \mathbb{R P} & \mathbb{P} \mathbb{R} & \mathbb{R} P & \mathbb{P R}\end{array}$ $\begin{array}{lllllllll}\mathbb{P} & \mathbb{R} & \mathbb{P} & \mathbb{R} & \mathbb{P} & \mathbb{P} & \mathbb{R} & \mathbb{R}\end{array}$

Here $\mathbb{P}$ refers to the Pomeron and $\mathbb{R}$ to any of $f_{2}, a_{2}, \omega$ and $\rho$ trajectories. A term $\left({ }_{3}^{1} 2\right)$ contributes to $d^{2} \sigma / d t d \xi$ as

$$
\begin{aligned}
& f_{1}(t) f_{2}(t) f_{3}(0) G_{3}^{12}(t) e^{i\left(\phi\left(\alpha_{1}(t)\right)-\phi\left(\alpha_{2}(t)\right)\right)} \xi^{1-\alpha_{1}(t)-\alpha_{2}(t)} \\
& \left(\frac{M_{X}^{2}}{s_{0}}\right)^{\alpha_{3}(0)-1} \cdot
\end{aligned}
$$

Here the $\alpha_{i}(t), i=1,2,3$ are the relevant Pomeron and Reggeon trajectories, $s_{0}$ is a scale factor that we take to be $1 \mathrm{GeV}^{2}$ and $\phi(\alpha(t))$ is the Reggeon phase. In practice only the first four terms of the series are required [33], those being $\left({ }_{\mathbb{P}}^{\mathbb{P}}\right),(\underset{\mathbb{R}}{\mathbb{P}}),(\underset{\mathbb{P}}{\mathbb{R}})$ and $(\underset{\mathbb{R}}{\mathbb{R}})$. Further it is sufficient to consider the $f_{2}, a_{2}, \omega$ and $\rho$ trajectories to be degenerate and use a single generic Reggeon exchange. The differential cross section may be written as

$$
\begin{aligned}
\frac{\partial^{2} \sigma}{\partial t \partial \xi}= & g_{\mathbb{P} \mathbb{P}}(t) s^{\alpha_{\mathbb{P}}(0)-1} \xi^{\alpha_{\mathbb{P}}(0)-2 \alpha_{\mathbb{P}}(t)} \\
& +g_{\mathbb{P} \mathbb{R}}(t) s^{\alpha_{\mathbb{R}}(0)-1} \xi^{\alpha_{\mathbb{R}}(0)-2 \alpha_{\mathbb{P}}(t)} \\
& +g_{\mathbb{R} \mathbb{R} P}(t) s^{\alpha_{\mathbb{P}}(0)-1} \xi^{\alpha_{\mathbb{P}}(0)-2 \alpha_{\mathbb{R}}(t)} \\
& +g_{\mathbb{R} \mathbb{R} \mathbb{R}}(t) s^{\alpha_{\mathbb{R}}(0)-1} \xi^{\alpha_{\mathbb{R}}(0)-2 \alpha_{\mathbb{R}}(t)}
\end{aligned}
$$

with

$g_{i i k}(t)=f_{i}(t)^{2} f_{k}(0) G_{k}^{i i}(t)$,

where $i$ and $k$ denote $\mathbb{P}$ or $\mathbb{R}$ as appropriate.

The Pomeron trajectory [33] is given by

$$
\begin{aligned}
\alpha_{\mathbb{P}}(t) & =1+\epsilon_{\mathbb{P}}+\alpha_{\mathbb{P}}^{\prime} t \\
& =1+0.08+0.25 t
\end{aligned}
$$

and

$$
\begin{aligned}
\alpha_{\mathbb{R}}(t) & =1+\epsilon_{\mathbb{R}}+\alpha_{\mathbb{R}}^{\prime} t \\
& =1-0.45+0.93 t
\end{aligned}
$$

is an "effective" reggeon trajectory, which is a reasonable average of the $f_{2}, a_{2}, \omega, \rho$ trajectories.

For the fitting procedure various parametrisations have been tested for the function $g_{i i k}(t)$,

$$
\begin{aligned}
& g_{i i k}(t)=A_{i} e^{B_{k} t} \\
& g_{i i k}(t)=A_{i} e^{B_{i} t}+C_{k} \\
& g_{i i k}(t)=A_{i} e^{B_{i} t+C_{k} t^{2}} \\
& g_{i i k}(t)=\lambda_{i}\left(\frac{t+A_{i}}{t+B_{i}}\right)^{C_{k}} F(t)^{2} .
\end{aligned}
$$

The most effective of these parametrisations, and the one we use here, is given by Eq. (32), where $A_{i}, B_{i}$ and $C_{k}$ are free parameters given by the fit to the data. The chosen parametrisation works perfectly at low energy but gives too high a cross section at $\sqrt{s}=546 / 640 \mathrm{GeV}$. For this reason the triple Pomeron coupling term $g_{\mathbb{P P P}}(t)$ is parametrized in a different way based on the possibility that it vanishes at $t=0[40]$

$$
\left(A_{i} e^{B_{i} t}+C_{k}\right)\left(\frac{t}{t+t_{0}}\right)
$$

where $t_{0}=-0.05 \mathrm{GeV}^{2}$ is the optimum value.

At small $t$, where the triple-Pomeron coupling term dominates, the vanishing term in the parametrisation improves the double differential cross section fit but it reduces the agreement of the differential cross section $d \sigma / d t$ with high-energy data. At the same time, at high energy and high $t$, the predicted differential cross section is lower than the data and this simple parametrisation fails. To improve the fit for both differential and double differential cross section the triple-Pomeron coupling parametrisation is divided into three different regions of $t$. For $-0.25 \leq t<-0.0001$ we use

$g_{\mathbb{P P P}}(t)=(0.4+0.5 t)$,

for $-1.15 \leq t<-0.25$ we use

$g_{\mathbb{P P P}}(t)=\left(A_{\mathbb{P}} e^{B_{\mathbb{P}} t}+C_{\mathbb{P}}\right)\left(\frac{t}{t-0.05}\right)$,

and for $-4.00 \leq t<-1.155$ we use

$$
\begin{array}{r}
g_{\mathbb{P} \mathbb{P}}(t)=\left(A_{\mathbb{P}} e^{B_{\mathbb{P}} t}+C_{\mathbb{P}}\right)\left(\frac{t}{t-0.05}\right) \\
\times\left(1+0.4597(|t|-1.15)+5.7575(|t|-1.15)^{2}\right) .
\end{array}
$$

We use a linear parameterisation at low $|t|$ to avoid unphysical behaviour and a modified form at high $|t|$ to increase the integrated cross section.

One additional term is required: a "Reggeized" pion exchange term which is important at low $t$ [33], this term (39) is kept fixed during the fitting procedure. In Regge theory the pion exchange term is given by

$\frac{\partial^{2} \sigma_{\pi}}{\partial t \partial \xi}=\frac{g_{\pi \pi p}^{2}}{16 \pi^{2}} \frac{|t|}{\left(t-m_{\pi}\right)^{2}} F^{2}(t) \xi^{1-2 \alpha_{\pi}(t)} \sigma_{\pi^{0} p}(s \xi)$ 
where $g_{\pi \pi p}^{2} / 4 \pi=14.4$ [41] is the on-mass-shell coupling, $m_{\pi}$ is the pion mass, $\alpha_{\pi}(t)=0.93\left(t-m_{\pi}^{2}\right)$ is the pion trajectory and $F^{2}(t)$ is the proton form factor. In Eq. (39), $\sigma_{\pi^{0} p}(s \xi)[\mathrm{mb}]$, denotes the pion-proton cross section, modeled by [41]

$\sigma_{\pi^{0} p}(s \xi)=13.63(\xi s)^{0.0808}+31.79(\xi s)^{-0.4525}$,

so the overall SD double differential cross for high missing mass can be written as:

$$
\begin{aligned}
\frac{\partial^{2} \sigma^{H M}}{\partial t \partial \xi}= & g_{\mathbb{P} \mathbb{P}}(t) s^{\alpha_{\mathbb{P}}(0)-1} \xi^{\alpha_{\mathbb{P}}(0)-2 \alpha_{\mathbb{P}}(t)} \\
& +g_{\mathbb{P P \mathbb { R }}}(t) s^{\alpha_{\mathbb{R}}(0)-1} \xi^{\alpha_{\mathbb{R}}(0)-2 \alpha_{\mathbb{P}}(t)} \\
& +g_{\mathbb{R} \mathbb{R} P}(t) s^{\alpha_{\mathbb{P}}(0)-1} \xi^{\alpha_{\mathbb{P}}(0)-2 \alpha_{\mathbb{R}}(t)} \\
& +g_{\mathbb{R} \mathbb{R} \mathbb{R}}(t) s^{\alpha_{\mathbb{R}}(0)-1} \xi^{\alpha_{\mathbb{R}}(0)-2 \alpha_{\mathbb{R}}(t)} \\
& +\frac{g_{\pi \pi p}^{2}}{16 \pi^{2}} \frac{|t|}{\left(t-m_{\pi}\right)^{2}} F^{2}(t) \xi^{1-2 \alpha_{\pi}(t)} \sigma_{\pi^{0} p}(s \xi) .
\end{aligned}
$$

The fitting procedure described in Sect. 4.3.

\subsection{Low mass: background and resonances}

The single-diffractive dissociation at low mass is a delicate issue in diffractive dissociation studies. A lot of $p p \rightarrow p X$ data in the resonance region is available at very low energy, much of which is not relevant, but some is used as a guide. Useful information to model the resonance region comes from data at $s=565 \mathrm{GeV}^{2}$ for $t=-0.05 \mathrm{GeV}^{2}$ [42], where the $d^{2} \sigma / d \xi d t$ are averaged over $1.5 \leq M_{X}^{2} \leq 2.5 \mathrm{GeV}^{2}$.

Both Pomeron and Reggeon exchange conserve helicity, so resonance excitation is primarily through incremental angular momentum with no change in the quark spin. On the basis of the Gribov-Morrison rule $[32,43]$ we expect the resonance to have spin-parity $(1 / 2)^{+},(3 / 2)^{-},(5 / 2)^{+}$, $(7 / 2)^{-}$etc. Also the dominant exchanges (Pomeron, $f_{2}, \omega$ ) are isoscalar, so we expect that the leading resonances produced are $P_{11}(1440), D_{13}(1520), F_{15}(1680)$ and $G_{17}(2190)$. The background to these leading resonances comes from the low-mass continuation of the high-mass model of Pomeron and Reggeon exchange.

Hadron-hadron scattering at low energies can be described by the sum of a few amplitudes for direct $s$-channel production; as the energy increases these resonances increasingly overlap and more amplitudes need to be considered. At high energies it can be described by the sum of a few simple Reggeon exchange amplitudes in the $t$-channel; as the energy falls more of these are required. The principle of duality asserts that these are two descriptions of the same physics, valid at lower and higher energies.

This can be extended to the principle of two component duality [41] in which the $s$-channel amplitudes comprise a smooth background which is dual to Pomeron exchange, and a set of resonances which is dual to Reggeon (non-Pomeron) exchanges.

We determine the background term first. We assume it is quadratic, unlike a previous analysis [42] which used a general polynomial, as this can match the triple-Regge form. The contribution vanishes at threshold, $\xi_{t h}=\left(M_{p}+m_{\pi}\right)^{2} / s$, and can therefore be written

$B(\xi, t, s)=a(t, s)\left(\xi-\xi_{t h}\right)^{2}+b(t, s)\left(\xi-\xi_{t h}\right)$.

$a$ and $b$ are then determined by requiring that the background matches smoothly onto the high mass region at some chosen value $\xi_{c}$ which represents the division between 'low' and 'high' mass.

Writing the triple-Regge function $\frac{\partial^{2} \sigma^{H M}}{\partial t \partial \xi}(\xi, t, s)$ as $A(\xi, t$, $s)$ the boundary conditions can be written as

$$
\begin{aligned}
A\left(\xi_{c}, t, s\right) & =B\left(\xi_{c}, t, s\right) \\
A^{\prime}\left(\xi_{c}, t, s\right) & =B^{\prime}\left(\xi_{c}, t, s\right)
\end{aligned}
$$

and the resulting background coefficients are given by

$$
\begin{aligned}
& a(t, s)=\frac{\left(\xi_{c}-\xi_{t h}\right) A^{\prime}\left(\xi_{c}, t, s\right)-A\left(\xi_{c}, t, s\right)}{\left(\xi_{c}-\xi_{t h}\right)^{2}} \\
& b(t, s)=2 \frac{A\left(\xi_{c}, t, s\right)}{\xi_{c}-\xi_{t h}}-A^{\prime}\left(\xi_{c}, t, s\right) .
\end{aligned}
$$

To complete the low mass model we now add the resonances contribution. Each baryon resonance is parametrised by a Breit-Wigner function, with a mass $m_{l}$ and a width $\gamma_{l}$. So the total contributions from resonances to the SD cross section at low-mass is given by

$\frac{d \sigma_{R e s}}{d M_{X}^{2}}=\sum_{l=1}^{4}\left[\frac{c_{l}}{M_{X}^{2}} \frac{m_{l} \Gamma_{l}}{\left(M_{X}^{2}-m_{l}^{2}\right)^{2}+\left(m_{l} \Gamma_{l}\right)^{2}}\right]$,

with

$\Gamma_{l}=\gamma_{l}\left(\frac{q}{q_{l}}\right)^{2 l+1}\left(\frac{1+5 q_{l}}{1+5 q}\right)^{l}$,

where $q$ and $q_{l}$ are respectively the 3-momenta at $M_{X}$ and $m_{l}$ in the resonance rest frame, assuming that $\pi p$ is the dominant final state. They are given by

$$
\begin{aligned}
& q\left(M_{X}^{2}\right)=\sqrt{\frac{\left(M_{X}^{2}-\left(M_{p}+M_{\pi}\right)^{2}\right)\left(M_{X}^{2}-\left(M_{p}-M_{\pi}\right)^{2}\right)}{4 M_{X}^{2}}} \\
& q_{l}=\sqrt{\frac{\left(m_{l}^{2}-\left(M_{p}+M_{\pi}\right)^{2}\right)\left(m_{l}^{2}-\left(M_{p}-M_{\pi}\right)^{2}\right)}{4 m_{l}^{2}}}
\end{aligned}
$$


The data from [42] for $t=-0.05 \mathrm{GeV}^{2}$ at $\sqrt{s}=23.7 \mathrm{GeV}$ are fitted as a sum of the background and these four leading resonances.

The $t$ dependence in the resonance region comes from Schamberger [42]. For $1.5 \leq M_{X}^{2} \leq 2.5 \mathrm{GeV}^{2}, d \sigma / d t \approx$ $\exp ((13.2 \pm 0.3) t)$. As there is some slight $t$-dependence in the background the double-differential cross section is obtained by multiplying Eq. (46) by $\exp (13.5(t+0.05))$ In terms of the variable $\xi$, this is

$$
\begin{aligned}
\frac{\partial^{2} \sigma_{R e s}}{\partial \xi \partial t}(\xi, t, s)= & e^{13.5(t+0.05)} \sum_{l=1}^{4} \\
& \times\left[\frac{c_{l}}{\xi} \frac{m_{l} \Gamma_{l}}{\left(\xi s-m_{l}^{2}\right)^{2}+\left(m_{l} \Gamma_{l}\right)^{2}}\right] .
\end{aligned}
$$

If the resonance contribution is simply added to the background there will be a small step between high and low mass regions at $\xi=\xi_{c}$. This is remedied by subtracting a small matching term $R_{m}$ linear in $\xi$, which is zero at threshold and equal to the magnitude of the resonance term at the matching point,

$R_{m}(\xi, t, s)=-\frac{\partial^{2} \sigma_{R e s}}{\partial \xi \partial t}\left(\xi_{c}, t, s\right) \frac{\xi-\xi_{t h}}{\xi_{c}-\xi_{t h}}$.

The total resonance contribution can then be written as

$R(\xi, t, s)=\frac{\partial^{2} \sigma_{R e s}}{\partial \xi \partial t}(\xi, t, s)+R_{m}(\xi, t, s)$,

and the complete single-diffractive double differential cross section at low mass is given by

$\frac{\partial^{2} \sigma^{L M}}{\partial t \partial \xi}(\xi, t, s)=R(\xi, t, s)+B(\xi, t, s)$.

\subsection{Fitting procedures}

The large amount of data on soft diffraction dissociation is given in 1 . It covers the ranges $17.2<\sqrt{s}<546 \mathrm{GeV}$ and $0.015<|t|<4.15 \mathrm{GeV}^{2}$, thus spanning the energies and the range of momentum transfer required. However there are clear inconsistencies of normalisation between different data sets and considerable variation in quality. In some data, for example from the ISR, the experimental resolution is insufficient to delineate clearly the resonance from the triple-Regge region, so fits to these data were restricted to $\xi>0.01$. The twelve parameters of the parametrisation of Eqs. (31)-(34), $g_{i i k}$, are obtained from a global fit over all the available data using MINUIT within ROOT [29].

Full systematic errors and correlations between experimental data sets are taken into account, and we consider two ways of doing this. The data are quoted with statistical and
Table 5 Fit parameters for the triple-Regge model

\begin{tabular}{lcll}
\hline Term & $A_{i}$ & $B_{i}$ & $C_{k}$ \\
\hline $\mathbb{P} \mathbb{P} P$ & 2.58 & 0 \\
$\mathbb{P} \mathbb{P}$ & 0.625 & 4.51 & 0.186 \\
$\mathbb{R} \mathbb{P}$ & 3.09 & 3.03 & 10.0 \\
$\mathbb{R} \mathbb{R} R$ & 4.00 & 5.86 & 21.0 \\
\hline
\end{tabular}

systematic errors. The former are due to Poisson statistics on the number of particles counted. The latter are dominated by uncertainties in the acceptance, and are common to all measurements made by a given experiment. These errors are strictly multiplicative but they are small enough in practice to be taken as additive, greatly simplifying the analysis.

If the systematic errors are not considered, the quantity to be minimised is

$\chi^{2}=\sum_{i} \sum_{j=1}^{N_{i}} \frac{\left(f_{i j}-d_{i j}\right)^{2}}{\sigma_{i j}^{2}}$,

where $i$ is the number of the experiment, $j$ the measurement within that experiment's dataset, and $f_{i j}, d_{i j}$ and $\sigma_{i j}$ are respectively the fitted function, the measured cross section, and the quoted statistical error.

If the experiment also quotes a systematic uncertainty on the acceptance of $\alpha_{i}$, so that each measurement has an error $S_{i j}=\alpha_{i} d_{i j}$, it is not possible to merely replace $\sigma_{i j}^{2}$ in the denominator by $\sigma_{i j}^{2}+S_{i j}^{2}$, because although this correctly expresses the variance of an individual point, it does not take into account the correlation between points. One way to allow for the correlations is to include them in the $\chi^{2}$ using the formula $\chi^{2}=\left(\mathbf{f}^{T}-\mathbf{d}^{T}\right) \mathbf{V}^{-1}(\mathbf{f}-\mathbf{d})$ where $\mathbf{V}$ is the covariance matrix for the measurements. This is included in the fitting procedure and gives reasonable results, although it is not clear what the fit is doing to the normalisations of the individual experiments.

An alternative approach is therefore taken, in which each experiment's results are adjusted by a factor $F_{i}$. These factors are included as parameters in the fit, with values initially set to 1 , and a contribution of $\frac{\left(F_{i}-1\right)^{2}}{\alpha_{i}^{2}}$ is added to the $\chi^{2}$ for each such term. The results of this procedure are very similar (and, for test cases, identical) and the fitted value of $F$ yielded useful information as to what the fit was doing to the normalisation of the individual experiments. $F_{i}$ values initially set to 1 have been found to vary between 0.9 and 1.15 which is within the $20-25 \%$ of quoted systematic errors between different experiments.

The minimisation is performed over 5562 data points yielding a $\chi^{2} / N D F=8.61$. The fit parameters are listed in Table 5 and the coefficients of the resonance contribution $c_{l}$ in the low mass region arising from the fit (we do not float 
Table 6 Resonance parameters

\begin{tabular}{lllll}
\hline Resonance & $l$ & $m_{l}[\mathrm{GeV}]$ & $\gamma_{l}$ & $c_{l}$ \\
\hline$P_{11}$ & 1 & 1.44 & 0.325 & 3.07 \\
$D_{13}$ & 2 & 1.52 & 0.130 & 0.415 \\
$F_{15}$ & 3 & 1.68 & 0.140 & 1.11 \\
$G_{17}$ & 4 & 2.19 & 0.450 & 0.952 \\
\hline
\end{tabular}

Table 7 The high mass diffractive fit experimental normalisation used

\begin{tabular}{ll}
\hline Experiment & Normalisation \\
\hline Albrow & 0.8698 \\
Armitage & 0.8956 \\
Schamberger & 1.0444 \\
Cool & 1.1111 \\
Akimov & 1.0629 \\
UA4 & 0.9775 \\
\hline
\end{tabular}

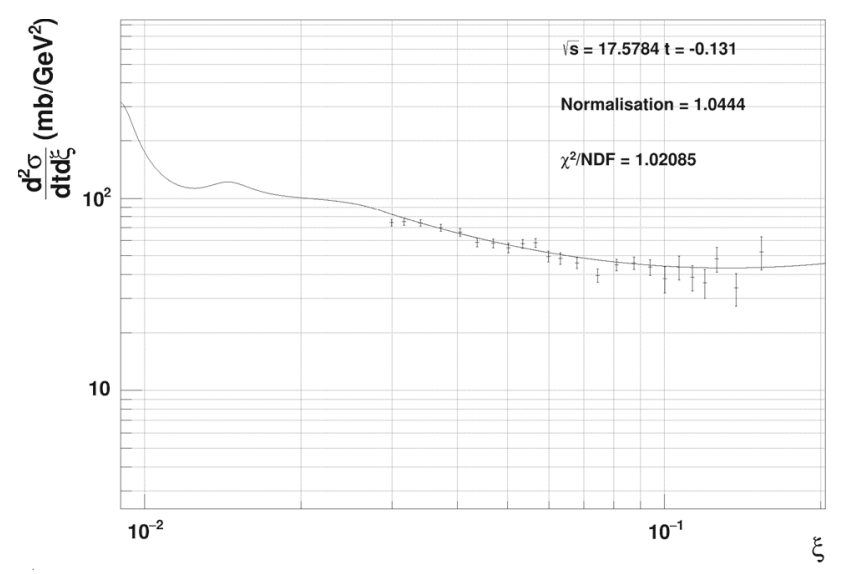

Fig. 8 The single diffraction DL model fit shown for $\sqrt{s}=17.57 \mathrm{GeV}$ and $t=-0.131 \mathrm{GeV}^{2}$

the resonance widths or locations) are given in Table 6). The normalisation for each experimental data set is presented in Table 7.

Typical fits are shown in Figs. 8 and 9 for the double differential cross section; other results at different momentum transfer $t$ and energies are reported in 1 .

In Fig. 10 we show the fit of $d^{2} \sigma^{L M} / d M_{X}^{2} d t$ to the data [42] at low mass. The background is the green line, the resonance structure is blue and the total in red. The resonance structure of the low mass region is reflected in the data with a strong peak at $P_{11}(1440)$ followed by a decreasing contribution from the remaining resonances. A comparison for the full range of the missing mass $M_{X}$ is presented in Fig. 11; the data at high mass are from [44].

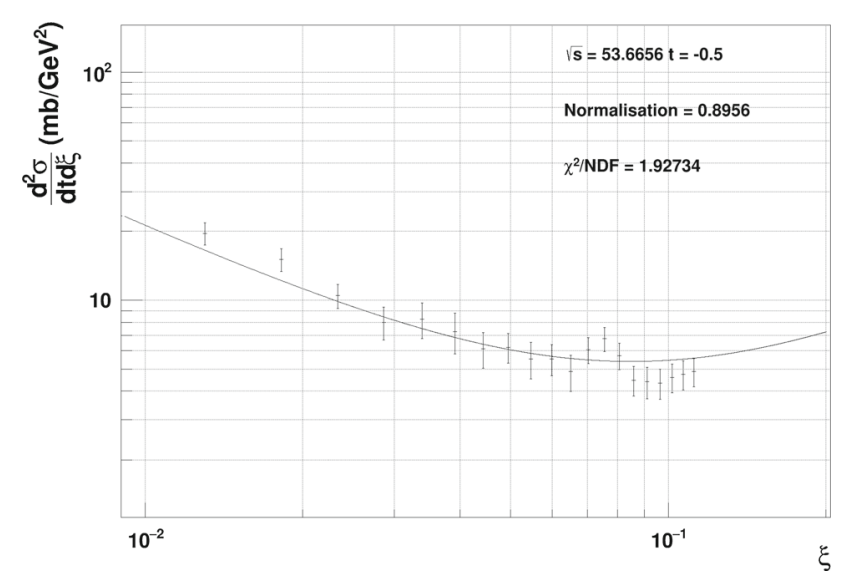

Fig. 9 The single diffraction DL model fit shown for $\sqrt{s}=53.66 \mathrm{GeV}$ and $t=-0.5 \mathrm{GeV}^{2}$

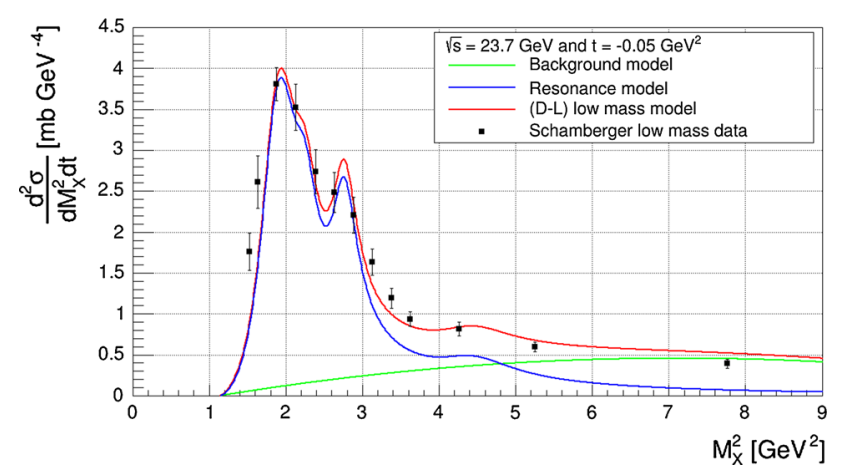

Fig. 10 Contribution to $d^{2} \sigma / d M_{X}^{2} d t$ from the background in blue, the resonance in red and the total in green. The black points represent data from Schamberger [42] for $t=-0.05 \mathrm{GeV}^{2}$ at $\sqrt{s}=23.7 \mathrm{GeV}$

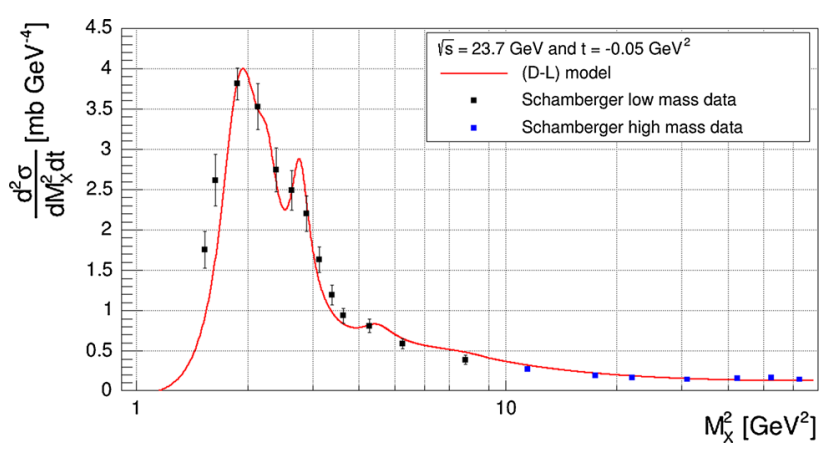

Fig. 11 Double differential cross section at $\sqrt{s}=23.7 \mathrm{GeV}$ and $t$ $=-0.05 \mathrm{GeV}^{2}$. The red line is the DL model and, black and blue points represent, respectively, the data at low [42] and high $M_{X}$ [44]

\subsection{Single differential SD cross section}

Data for the single differential SD cross section, $d \sigma / d t$ after integration over $\xi$, are available for a large range of energies and are used to compare the fit results and as a guide in the fitting procedure of the double differential cross section data. 


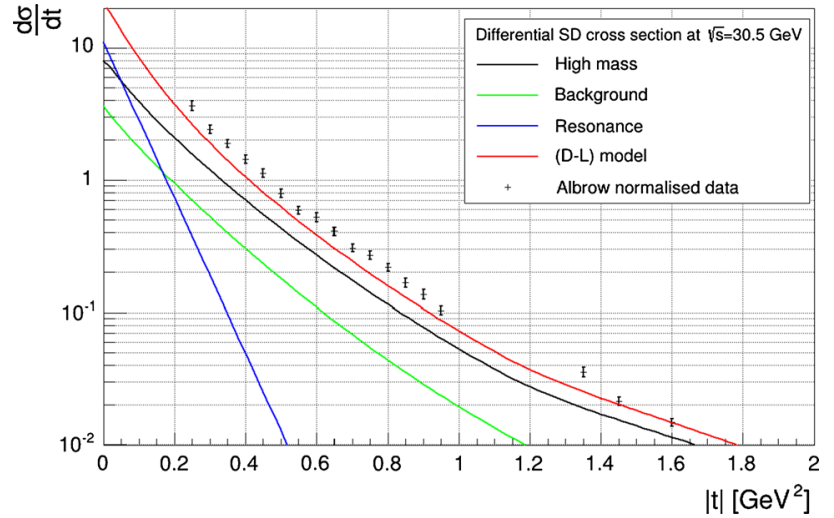

Fig. 12 The single diffraction differential cross section at $\sqrt{s}=30.5$ $\mathrm{GeV}$. In blue the resonance, in green the background and in black the high mass contribution to the differential cross section. The total DL model is shown in red. The black points represent data from Albrow

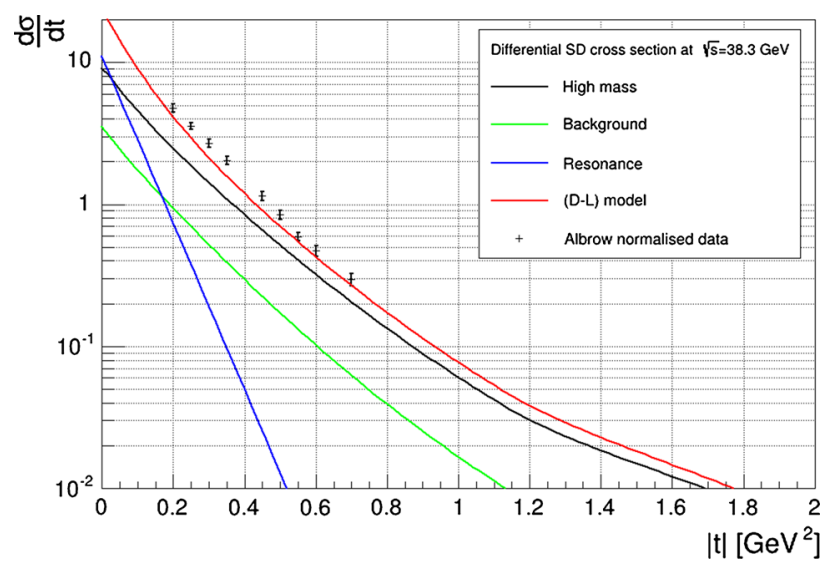

Fig. 13 The single diffraction differential cross section at $\sqrt{s}=38.3$ $\mathrm{GeV}$. In blue the resonance, in green the background and in black the high mass contribution to the differential cross section. The total DL model is shown in red. The black points represent data from Albrow

Some examples are shown in Figs. 12 and 13 for low energies, $\sqrt{s}=30.5$ and $38.3 \mathrm{GeV}$, and Fig. 14 for high energy UA4 data [45]. We show in blue the resonance contribution, in green the background and in black the high mass contribution to the differential cross section. The total DL model is shown in red.

The DL model at low energies does not match the experimental data perfectly, due to an underestimated contribution from the resonances. At low $t$ there is a strong contribution to the differential cross section from resonances and background, for higher $t$ the high mass term dominates. The effect of the correction at low $t$ from the triple-Pomeron term of the high mass contribution is visible, for example, in Fig. 14 where the DL model matches the data very well. The modification at high $t$ is a reasonable compromise at low and high energies.

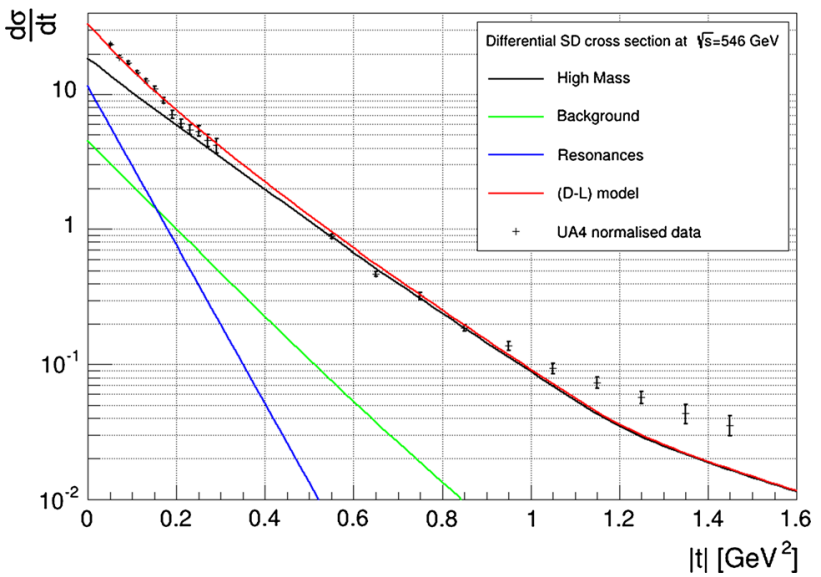

Fig. 14 The single diffraction differential cross section at $\sqrt{s}=546$ $\mathrm{GeV}$. In blue the resonance, in green the background and in black the high mass contribution to the differential cross section. The total DL model is shown in red. The black points represent data from UA4 collaboration

\subsection{Integrated SD cross section $s$ dependence}

The energy dependence of the total SD cross section is a controversial topic. For energy $(\sqrt{s})$ below $25 \mathrm{GeV}$ the standard Regge theory reproduces the SD cross section well, however it rises faster than the experimental observations at higher energy. This behaviour was already expected theoretically due to problems related with the violation of the unitarity at high energy, i.e. $\sigma_{S D}>\sigma^{\text {tot }}$ and the Froissart bound [46]. Some different theoretical approaches have been attempted to overcome this problem, including the renormalisation of standard Pomeron flux to agree with the data [47] or decoupling of the triple Pomeron vertex [48].

Single-diffraction data at $\sqrt{s}=7 \mathrm{TeV}$ have been obtained by ALICE [49], CMS [50] and TOTEM [51]. The ALICE data are consistent with the integrated single-diffraction cross section increasing with energy. In contrast the CMS and TOTEM integrated cross sections, for $8<M_{X}<350$ $\mathrm{GeV}$ and $6.5<M_{X}<1100 \mathrm{GeV}$ respectively, appear to show a decrease with increasing energy when compared to extrapolations of conventional models. For $M_{X}<3.4 \mathrm{GeV}$ TOTEM [52] give an integrated cross section of ( $2.2 \pm 2.17)$ $\mathrm{mb}$ and an upper limit of $6.31 \mathrm{mb}$ at $95 \%$ confidence level which is not inconsistent with the UA4 value of $3.0 \pm 0.8$ $\mathrm{mb}$ at $\sqrt{s}=546 \mathrm{GeV}$ and still allows, in principle, some increase with energy.

A full discussion of the problem and possible modifications to the models is given in [53]. Including sophisticated modifications in our fitting of multiple data sets is impractical, so we adopted the simple alternative of modifying the chosen matching point $M_{c}=\sqrt{s} \xi_{c}$. We do not include data above $\sqrt{s}=546 / 630 \mathrm{GeV}$ and ensure agreement with the UA4 integrated cross sections [45] and $d \sigma / d t$. This is achieved with a parametrisation given by 
Table 8 DL model prediction and UA4 data for low-high mass and total SD cross section at $\sqrt{s}=546 \mathrm{GeV}$

\begin{tabular}{llll}
\hline Source & $\begin{array}{l}\sigma_{S D}^{\text {expt }}[\mathrm{mb}] \\
M_{X}<4 \mathrm{GeV}\end{array}$ & $\begin{array}{l}\sigma_{S D}^{\text {expt }[\mathrm{mb}]} \\
M_{X}>4 \mathrm{GeV}\end{array}$ & $\begin{array}{l}\sigma_{S D}^{\text {expt }}[\mathrm{mb}] \\
\xi<0.05\end{array}$ \\
\hline DL model & 2.89 & 6.59 & 9.485 \\
UA4 data & $3 \pm 0.8$ & $6.4 \pm 0.4$ & $9.4 \pm 0.4$ \\
\hline
\end{tabular}

$$
M_{c}(s)= \begin{cases}3 & \text { for } s<s_{0} \\ 3+\alpha \ln \left(\frac{s}{s_{0}}\right) & \text { for } s>s_{0},\end{cases}
$$

where $\alpha=0.6 \mathrm{GeV}$ and $s_{0}=4000 \mathrm{GeV}^{2}$.

A comparison between our model and the UA4 results is presented in Table 8, with the experimentally determined SD cross section $\sigma_{S D}^{\text {expt }}=2 \sigma_{S D}$, where $\sigma_{S D}$ is the integrated cross section, to take into account both arms of the SD diagram as usually quoted by experimentalists. The agreement between the data and the integrated model is very good. The total integrated single diffractive cross section, integrated up to $\xi<0.05$, is shown in Fig. 15 . The red line is the DL model and the experimental points are indicated with their normalisations. The vertical dashed blue lines represents the energy range of the LHC.

The model works well in this region, and some distance beyond. At very low energy the Regge model does not accurately describe the data, which is dominated by $s$-channel resonances, but this is well below our region of applicability.

\subsection{Application of the model at the LHC energies}

For the LHC collimation studies the range of centre-of-mass energy $\sqrt{s}$ is between $81-115 \mathrm{GeV}$ for collisions. We use our
Table 9 The single diffractive cross section at LHC energies. The cross section unit are mb

\begin{tabular}{llll}
\hline $\mathrm{E}[\mathrm{GeV}]$ & $\sqrt{s}[\mathrm{GeV}]$ & $\sigma_{S D}(\xi<0.05)$ & $\sigma_{S D}(\xi<0.12)$ \\
\hline 3500 & 81 & 3.39 & 4.37 \\
7000 & 115 & 3.55 & 4.53 \\
\hline
\end{tabular}

model to predict the total and double differential SD cross section at LHC energies. The single diffractive cross sections are reported in Table 9 for $\xi<0.05$ and $\xi<0.12$, the first is given as reference because it is the upper limit used in many publications on the subject and the second to present the values that we are using in our model to predict the LHC loss maps.

Figure 16 shows the contribution of the background and resonances to the double differential cross section at $s=$ $114^{2} \mathrm{GeV}^{2}$ for different values of the transfer momentum $t$ (from left to right $t=-0.01, t=-0.4$ and $t=-2 \mathrm{GeV}^{2}$ ). The blue line represents the resonance contribution, the red line the background contribution and the green line the total low mass fit. The black line represents the triple-Regge fit at high mass. The contributions of resonances and background are stronger at low $t$ and decrease at medium $t$; for high $t$ the contribution from resonances disappears and the cross section is dominated by the background.

For the high mass region the contribution of the leading trajectories and the pion-exchange term are shown in Fig. 17 for different value of the momentum transfer $t$. The plot on the left shows the contributions for low $t$, the triple Pomeron term, $\mathbb{P P P}$ in red, and the $\mathbb{P P R}$ term in blue, dominate at low $\xi$, with some contribution from $\mathbb{R} \mathbb{R} \mathbb{P}$ in green and $\mathbb{R} \mathbb{R}$ in cyan. At higher $\xi$ the main contribution to the sum
Fig. 15 Total integrated SD cross section $\sigma_{S D}^{\text {tot }}$ at different energies. The red line represents the DL model results and the points are the experimental data reported with their normalisation. The vertical dashed blue lines represent the energies range of interest for the LHC

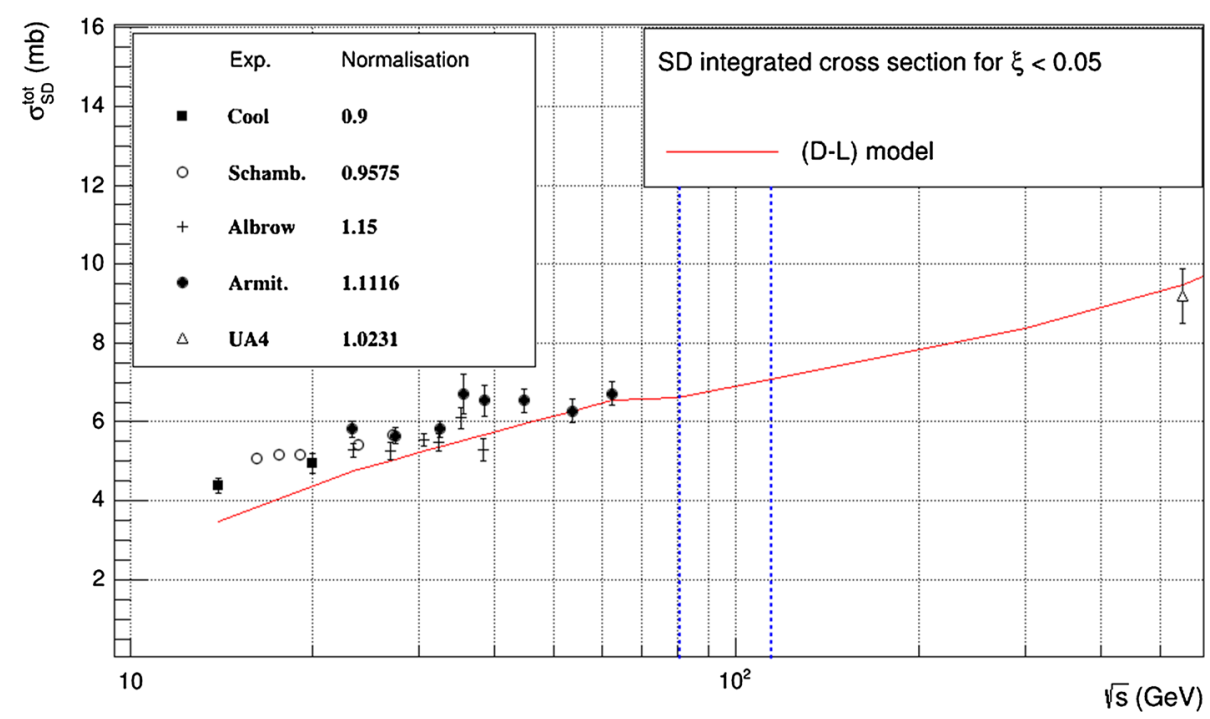




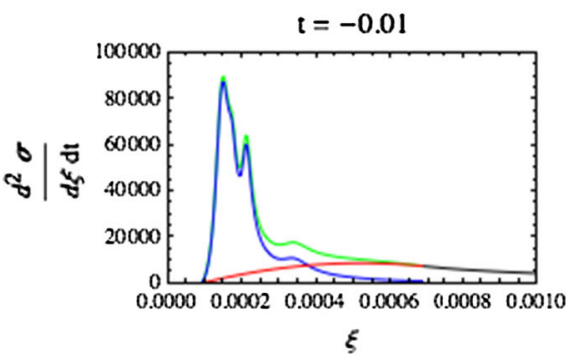

Fig. 16 Individual contributions for the low mass SD double differential cross section for the LHC at top energy, $s=114.6^{2} \mathrm{GeV}^{2}$, from left to right $t=-0.01, t=-0.4$ and $t=-2 \mathrm{GeV}^{2}$. In blue the resonance function $R\left(\xi, t, s_{L H C}\right)$, in red the background $B\left(\xi, t, s_{L H C}\right)$ and
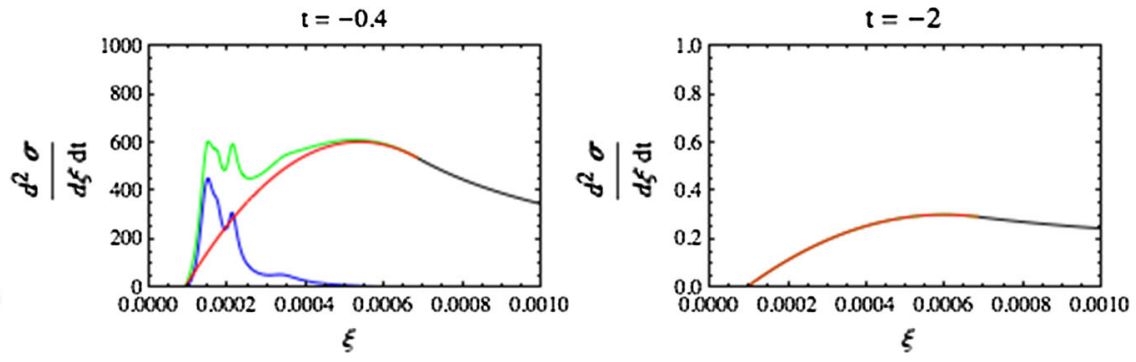

in green the total SD double differential cross section at low mass. The black line represents the triple-Regge fit at high mass, note that it goes up to $\xi=0.12$
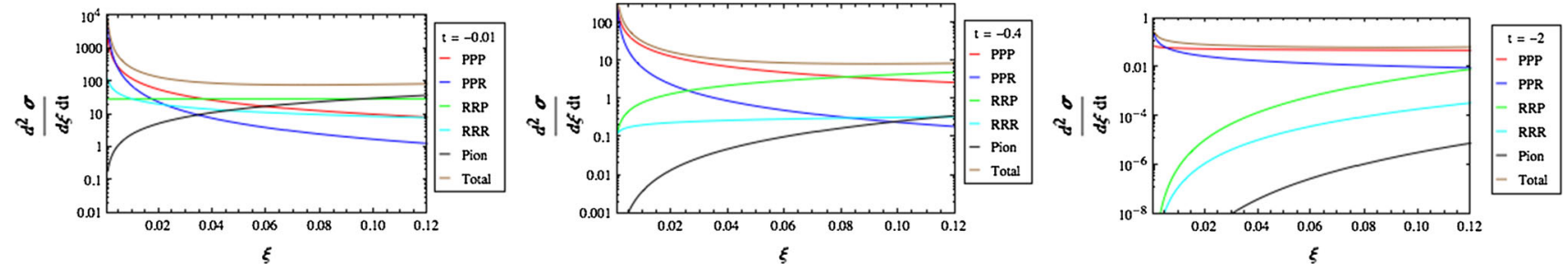

Fig. 17 Individual contributions of triple-Reggeon exchanges and pion exchange for the SD double differential cross section for the LHC at top energy, $s=114.6^{2} \mathrm{GeV}^{2}$, from left to right $t=-0.01, t=-0.4$ and $t=-2 \mathrm{GeV}^{2}$

of all terms, in brown, is given by the $\mathbb{R R P}$ and the pionexchange term in black. At medium and high $t$ the SD double differential cross section is dominated by the triple-Pomeron $\mathbb{P} \mathbb{P}$ term with some slight contribution from the remaining terms.

\section{Simulation of LHC loss maps using MERLIN}

We have incorporated the DL model, with the fitted parameters described in the previous sections, into the simulation code MERLIN, a C ++ accelerator physics library [13] which has been extended to include proton collimation [1012] with the aim of providing an accurate simulation of the Large Hadron Collider (LHC) collimation system. Up to now, the FORTRAN program SixTrack has been the main tool used to calculate dynamical aperture in the LHC, and, incorporating the scattering model known as K2 [54], has been used for many studies of the LHC collimation system and resulting the loss maps $[4-7,55]$. However a second program was felt to be desirable, as an independent check and to provide a more flexible and future-proof design to which new features, such as this model, can readily be added. The modular nature of MERLIN allows one to easily switch between the K2 scattering model and the DL model. As well as these nuclear processes, MERLIN also includes Coulomb scattering and other EM effects of protons in matter. The relative importance of these is discussed in [25] and will be presented in a future paper on the MERLIN code.

MERLIN was used to simulate the nominal optics at $7 \mathrm{TeV}$ of the LHC in order to generate loss maps, using a thick lens tracking model. These can be generated for different optics configurations, e.g. the $\beta$-function at the interaction points $\left(\beta^{*}\right)$ and beam crossing angles. As a first step, we benchmark its prediction of the optical functions against the MAD-X program [56], with the excellent agreement shown in Fig. 18.

We then, using the same scattering physics models, benchmarked it against the standard SixTrack $+\mathrm{K} 2$ predictions for the loss map calculation [5,12,57]. These studies have demonstrated the quantitative agreement between the established tools for loss map analysis and MERLIN.

The loss maps are characterised by the local inefficiency

$\eta=\frac{N_{A B S}}{\Delta z \cdot N_{\text {coll }}^{\text {tot }}}$,

which is plotted as a function of $s$, the distance around the ring. Here $\Delta z$ is the longitudinal resolution $(10 \mathrm{~cm}), N_{A B S}$ is the number of particles absorbed in $\Delta z$ and $N_{\text {coll }}^{\text {tot }}$ is the total loss in the collimators along the whole machine. For the collimator $\Delta z$ is set to the collimator length and $N_{A B S}$ are the total losses in the collimator.

In these simulations, if a proton undergoes an inelastic interaction inside the collimators, or if it touches the beam 
Fig. 18 The difference of the optical $\beta$-functions for the nominal LHC between MADX [56] and MERLIN

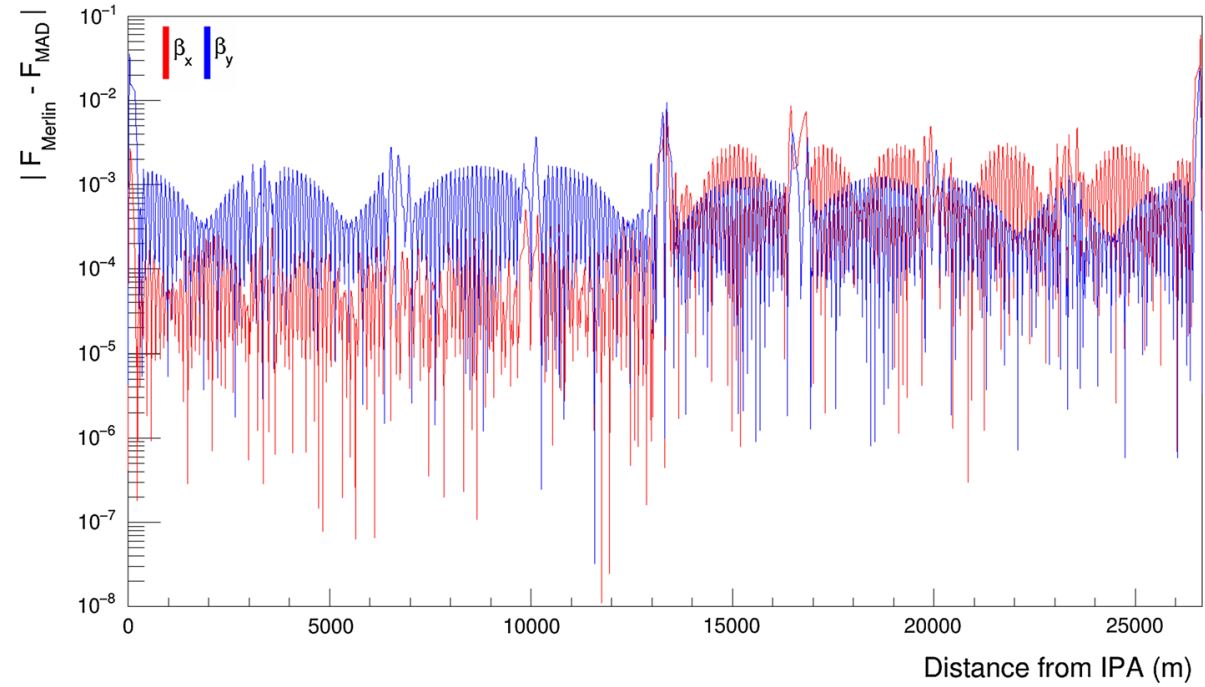

pipe, it is considered lost: the particle is removed from the bunch and the location at which this takes place is recorded, using a default bin size of $10 \mathrm{~cm}$. This is necessary as these conventions are those used by existing studies [4-7,55]; against which we benchmark the new code. They mark a sensible line between the study of long-distance (multi-turn) effects, which are the subject of simulations such as these, and short range effects which are the subject of programs like FLUKA [58] and GEANT4 [59]. When short-range effects are also needed, for example in the simulation of beam loss monitor responses, the lost particles are saved to a file and the shower evolution studied by one of these more detailed codes.

The main parameters for the loss map calculation are summarised in Table 10 for a squeezed beam with an IP beam separation and crossing angle applied. The beam impinges on the horizontal primary collimators (TCP.C6L7) in IR7, and is tracked for 200 turns. The transverse offset between the jaw surface and the impact point, called the impact parameter, is set to $1 \mu \mathrm{m}$.

The loss map calculated for this machine configuration and the DL scattering models is shown in Fig. 19. The plot is colour coded: black spikes represent losses in the collimator jaws, red spikes losses in warm elements of the accelerator, and most importantly blue spikes which indicate losses in the superconducting magnets. Using $64 \mathrm{M}$ simulated protons, MERLIN calculates a total loss inefficiency of $77.65 \%$, with $0.010 \%$ lost in cold regions and $0.011 \%$ in warm elements. The remaining protons are lost in the collimators.

The highest black peak in the map corresponds to the horizontal primary collimator in IR7, which has an aperture of 6 $\sigma$. This is the tightest aperture in the machine. The loss map has been compared to those computed with Sixtrack+K2 [3], and has been found to be consistent $[11,57]$ with most of the
Table 10 A list of the relevant parameters required for the loss maps simulation. The LHC optics sequence is the version V6.503 for beam 1

\begin{tabular}{ll}
\hline Parameter & Value \\
\hline Energy & $7 \mathrm{TeV}$ \\
Norm. Emittance $\epsilon_{n}$ & $3.75 \mathrm{~mm} \mathrm{mrad}$ \\
$\beta^{*}($ IR1 \& IR5) & $0.55 \mathrm{~m}$ \\
$\beta^{*}$ (IR2 \& IR8) & $10 \mathrm{~m}$ \\
Crossing angle (IR1) & $-145 \mu \mathrm{rad}$ \\
Crossing angle (IR5) & $145 \mu \mathrm{rad}$ \\
Crossing angle (IR2) & $-90 \mu \mathrm{rad}$ \\
Crossing angle (IR8) & $-220 \mu \mathrm{rad}$ \\
Longitudinal resolution & $10 \mathrm{~cm}$ \\
Turn number & 200 \\
\hline
\end{tabular}

losses in the dedicated collimation regions IR7 and IR3, and black peaks in the tertiary collimators used to protect the high luminosity insertion in IR1 (ATLAS ) and IR5 (CMS). The peaks downstream of IR7 and in the arcs IR7-IR1 and IR1IR2 are particularly important because they show where halo protons touch the SC dipoles. These predictions allow us to understand where possible quenching events may occur and how to optimise the collimation system.

The losses in the dispersion suppressor region of IR7 are shown in Fig. 20 along with the horizontal dispersion. The highest beam losses per unit length are in the primary collimators, followed by lower losses in the secondary and tertiary collimators. Between $19700 \mathrm{~m}$ and $20200 \mathrm{~m}$ there are not only losses on the collimators, appearing as sharp spikes with an efficiency $10^{-4} \mathrm{~m}^{-1}$ or above, but also regions with efficiencies around $10^{-5}$ to $10^{-6} \mathrm{~m}^{-1}$ in the warm magnets between the collimators; these are mainly single diffracted 
Fig. 19 Loss map result for beam 1 with DL scattering physics. The beam is impinged on the primary collimator TCP.C6L7 in IR7 region, corresponding to the highest peak in the plot. The black peaks correspond to the losses in the collimator elements, the red ones to losses in the warm elements and the blue ones are the losses in the cold magnetic elements of the machine

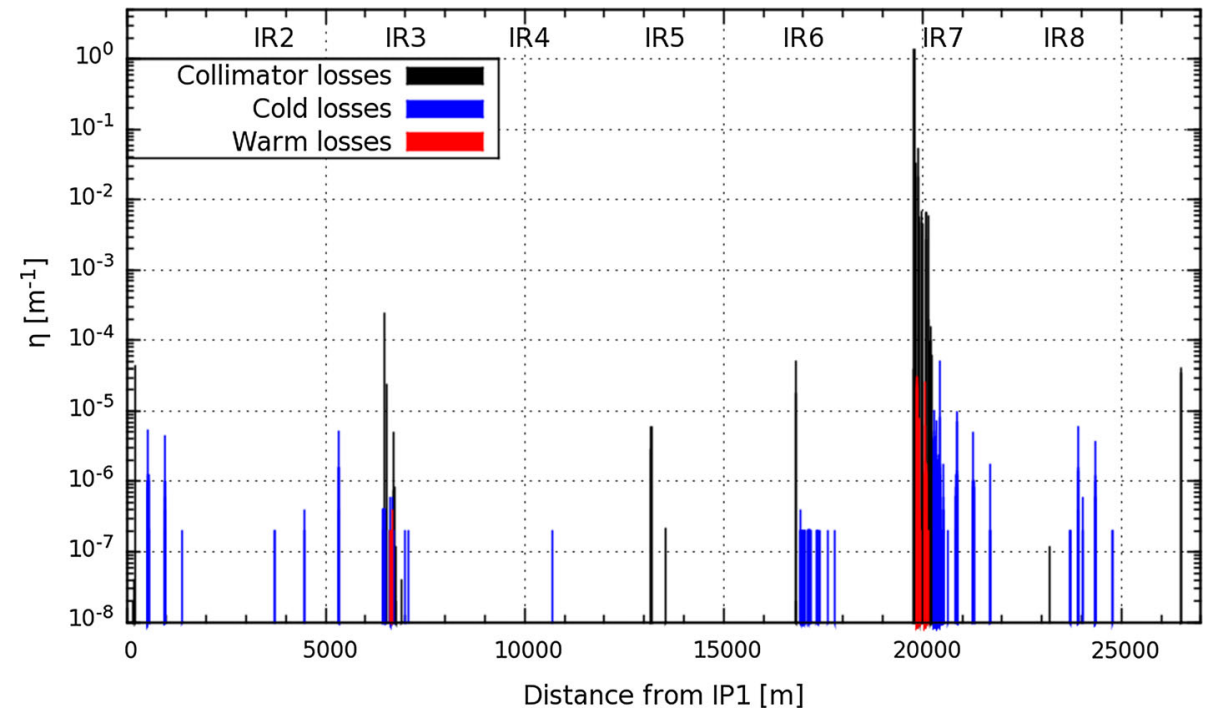

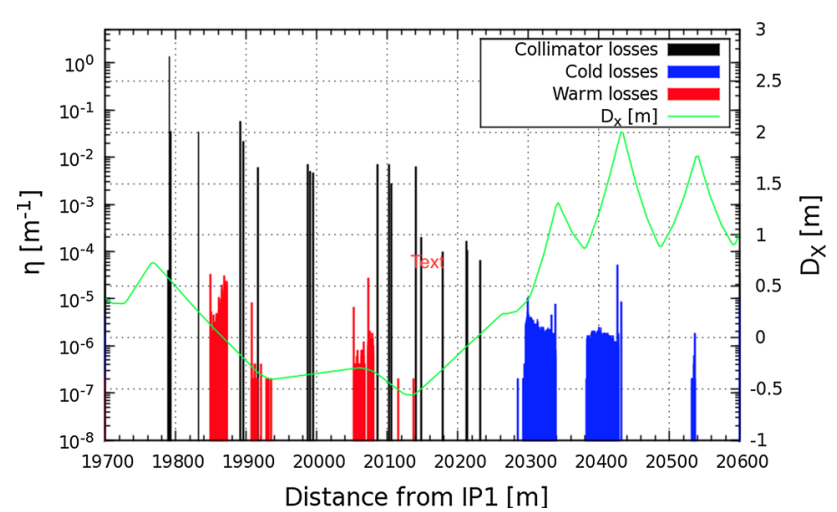

Fig. 20 Loss map in the betatron collimation region IR7. The local maxima of the dispersion (green line) are linked to the losses between $20300 \mathrm{~m}$ and $29400 \mathrm{~m}$, which is in a cold region. The black peaks correspond to the losses in the collimator elements, the red ones in the warm elements and the blue one are the losses in the cold magnetic element of the machine

protons with high momentum losses and scattered at high angles. MERLIN predicts more losses in this region than the K2 scattering routine [12] which has a different description of diffractive scattering. Losses downstream of IR7 in the dispersion suppressor, which are particularly sensitive areas, are shown in blue. Protons which experience single diffractive scattering in the bulk material of the collimator emerge with a transverse kick and a lower energy, so protons entering the dispersion suppressors, where the dispersion rises rapidly, can be lost in these cold areas. Most of the peaks in the cold part of the arc between IR7/IR8 are located at the local maxima of the dispersion, as shown for the first peak downstream of the dispersion suppressor in Fig. 20.

In Fig. 21 we present the distribution of energy lost $|\delta p / p|$ by protons impacting on the warm elements among

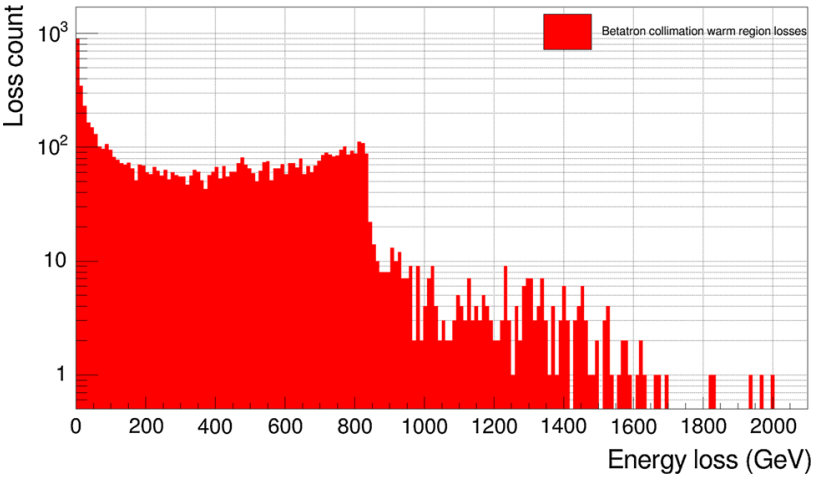

Fig. 21 Distributions of the $\delta p / p$ of the particles lost in the warm elements among collimators in IR7

the collimators in IR7. The plot shows a peak at low lost energy followed by a plateau till $10 \%$ and a rapid drop off. Figure 22 shows the distribution of energy lost in the dispersion suppressors (DS1, DS2) and particles lost downstream of IR7. The range of energy lost is between $2-10 \%$ for the first dispersion suppressor and $1-2 \%$ for the second.

\section{Conclusion}

We have presented a development of the model of Donnachie and Landshoff for elastic and single-diffractive proton scattering for use in simulating collimation systems in high energy proton accelerators. The model includes a description of elastic scattering combining Coulomb with Regge exchange amplitudes, and a description of diffractive scattering that combines $s$-channel resonance formation with $t$ channel Regge exchange. It is valid over a wide range in the centre of mass energy $\sqrt{s}$, the invariant 4-momentum 


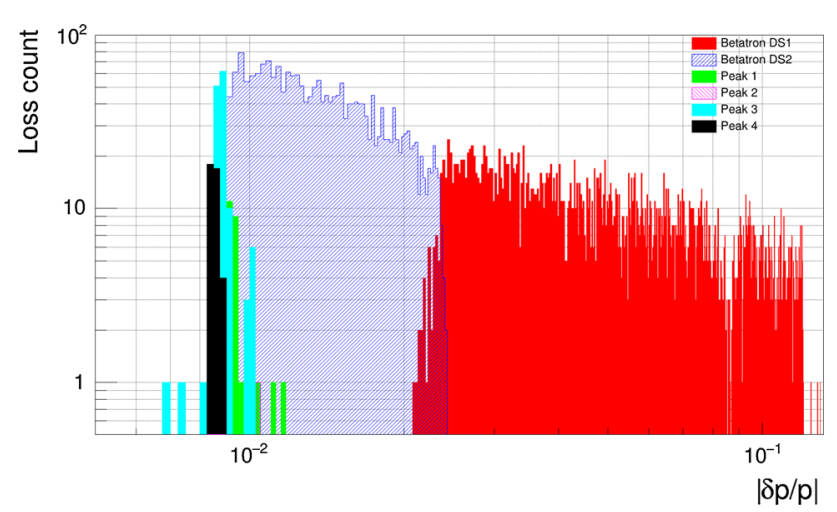

Fig. 22 Distributions of the $\delta p / p$ of the particles lost in the main peaks downstream IR7, grouped by dispersion suppressors and following cold peaks

transfer $\sqrt{t}$ and the scaled missing mass $\xi$, covering the relevant kinematical regions for the LHC (including the proposed high luminosity upgrade) and the Future Circular Collider.

We have taken elastic and diffractive scattering data from a large number of previous experiments, with different systematic errors, and fitted them with a small number of model parameters. The results have been incorporated into the MERLIN tracking code and this has been used to predict loss maps for $7 \mathrm{TeV}$ running at the LHC, as a first contribution to a future study of the collimation system in the LHC and proposed High-Luminosity upgrades, where it can contribute significantly to the design of the necessary improvements to the collimation system. The model includes important physics in an effective way, which has been included in a practical simulation program with the potential of understanding and improving the performance of collimation systems at present and future high energy proton accelerators.

Acknowledgments The authors wish to thank Prof. Donnachie for his support, for assisting us during the development of the scattering models and for being always available to discuss and improve the work. In particular he was responsible for the form of the model in the low mass region of the diffractive scattering. We also thanks Stefano Redaelli and Roderick Bruce for providing support for calculations relating to the LHC collimation system. The HiLumi LHC Design Study is included in the High Luminosity LHC project and is partly funded by the European Commission within the Framework Programme 7 Capacities Specific Programme, Grant Agreement 284404.

Open Access This article is distributed under the terms of the Creative Commons Attribution 4.0 International License (http://creativecomm ons.org/licenses/by/4.0/), which permits unrestricted use, distribution, and reproduction in any medium, provided you give appropriate credit to the original author(s) and the source, provide a link to the Creative Commons license, and indicate if changes were made.

Funded by SCOAP 3 .

\section{Appendix A Elastic data sources and model fit}

The elastic data sources with their energies and references are reported in Table 11. The normalisations chosen by the fitter can be found in [25]. In Fig. 23 we present the $p p$ elastic scattering model fit (fitted over all data) shown for a range of $\sqrt{s}$, over the full $t$ range, including the Coulomb peak and down to the lower cut on $t$, with the corresponding fits for $p \bar{p}$ shown in Fig. 24.

\section{Appendix B Single diffraction dissociation data sources and model fit}

The single diffraction dissociation data sources with their energies and references are reported in Table 12. The complete set of model fit comprise more than 300 plots. In Figs. 25 and 26 we present a selection of these plots to cover the full range of energies $(\sqrt{s})$ and $\xi$ and for low and high momentum transfer $t$.

Elastic and inelastic data has recently been published at LHC energies [88-91] . This would provide an interesting further study, but is not relevant to the relevant kinematic ranges of $t$ and $\xi$, as considered in this paper.

Table 11 A list of elastic data used in the fit and its source

\begin{tabular}{|c|c|c|c|}
\hline $\begin{array}{l}\text { Accelerator } \\
\text { (experiments) }\end{array}$ & Particles & $\sqrt{s}(\mathrm{GeV})$ & Sources \\
\hline ISR & $p p$ & 23.46 & {$[17,28,60,61]$} \\
\hline $\begin{array}{l}\text { Fermilab } \\
\text { (E177A) }\end{array}$ & $\mathrm{pp}$ & 27.426 & {$[62]$} \\
\hline ISR & $p p$ & 30.54 & {$[28,60,61,63]$} \\
\hline ISR & $p p$ & 44.64 & {$[60,64]$} \\
\hline ISR (R211,SFM) & $\mathrm{pp}$ & 52.81 & {$[28,60,63,65,66]$} \\
\hline ISR & $p p$ & 62.5 & {$[17,28,60,63]$} \\
\hline LHC (TOTEM) & $\mathrm{pp}$ & 7000 & {$[67,68]$} \\
\hline ISR & $p \bar{p}$ & 30.4 & {$[28,63]$} \\
\hline ISR & $p \bar{p}$ & 52.6 & {$[28,63,65,66]$} \\
\hline ISR & $p \bar{p}$ & 62.3 & {$[28,63]$} \\
\hline SPP̄S (UA4/2) & $p \bar{p}$ & 541 & [69] \\
\hline $\begin{array}{l}\text { SPP̄S (UA4), } \\
\text { Tevatron (CDF) }\end{array}$ & $p \bar{p}$ & 546 & {$[70-74]$} \\
\hline SPP̄S (UA4) & $p \bar{p}$ & 630 & [75] \\
\hline $\begin{array}{l}\text { Tevatron (CDF, } \\
\text { E710) }\end{array}$ & $p \bar{p}$ & 1800 & {$[74,76]$} \\
\hline Tevatron $(\mathrm{D} \varnothing)$ & $p \bar{p}$ & 1980 & [77] \\
\hline
\end{tabular}


520 Page 18 of 23

Eur. Phys. J. C (2016) 76:520
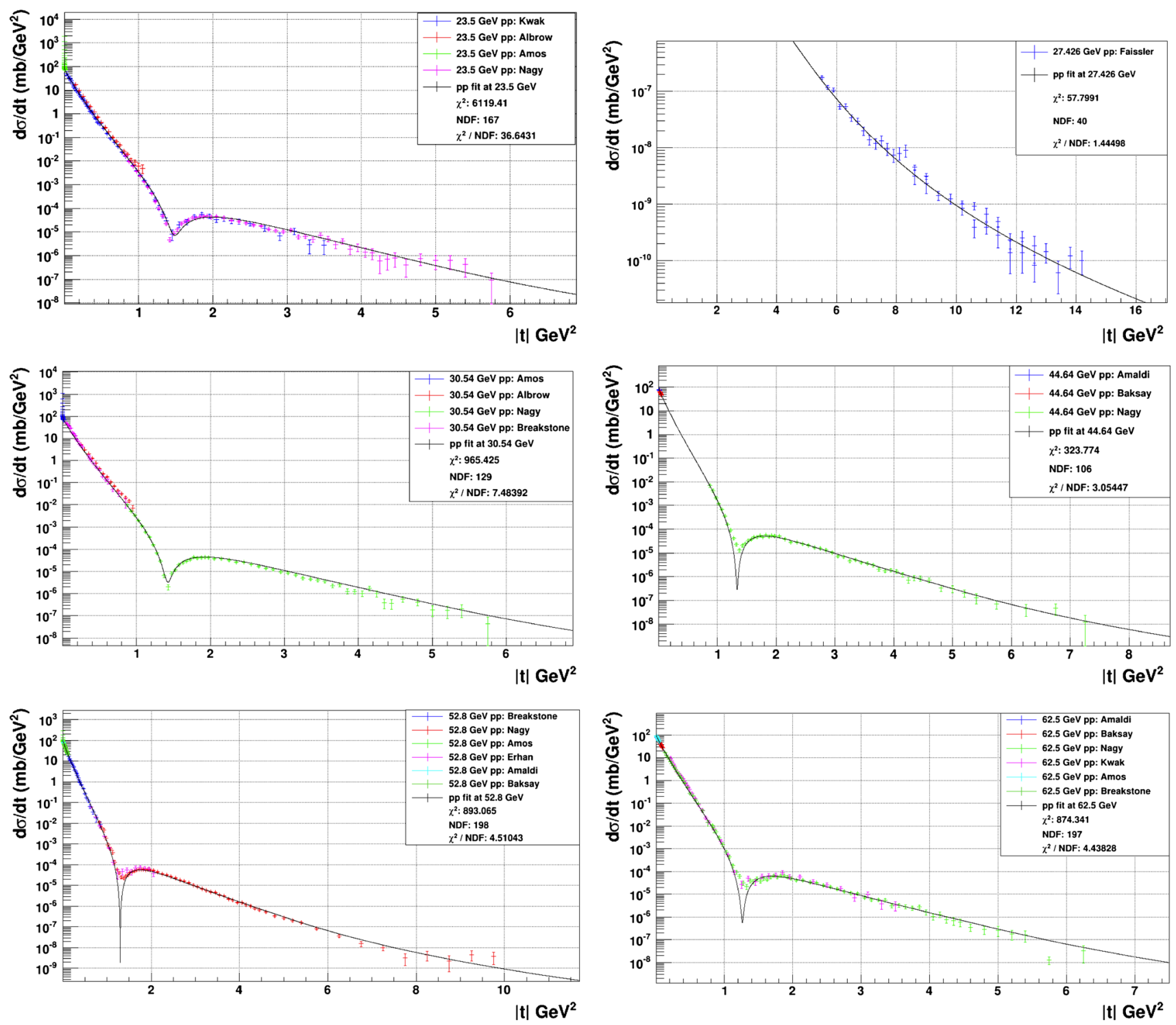

Fig. 23 The $p p$ elastic scattering model fit (fitted over all data) shown for a range of $\sqrt{s}$, over the full $t$ range, including the coulomb peak and down to the lower cut on $t$

Springer 

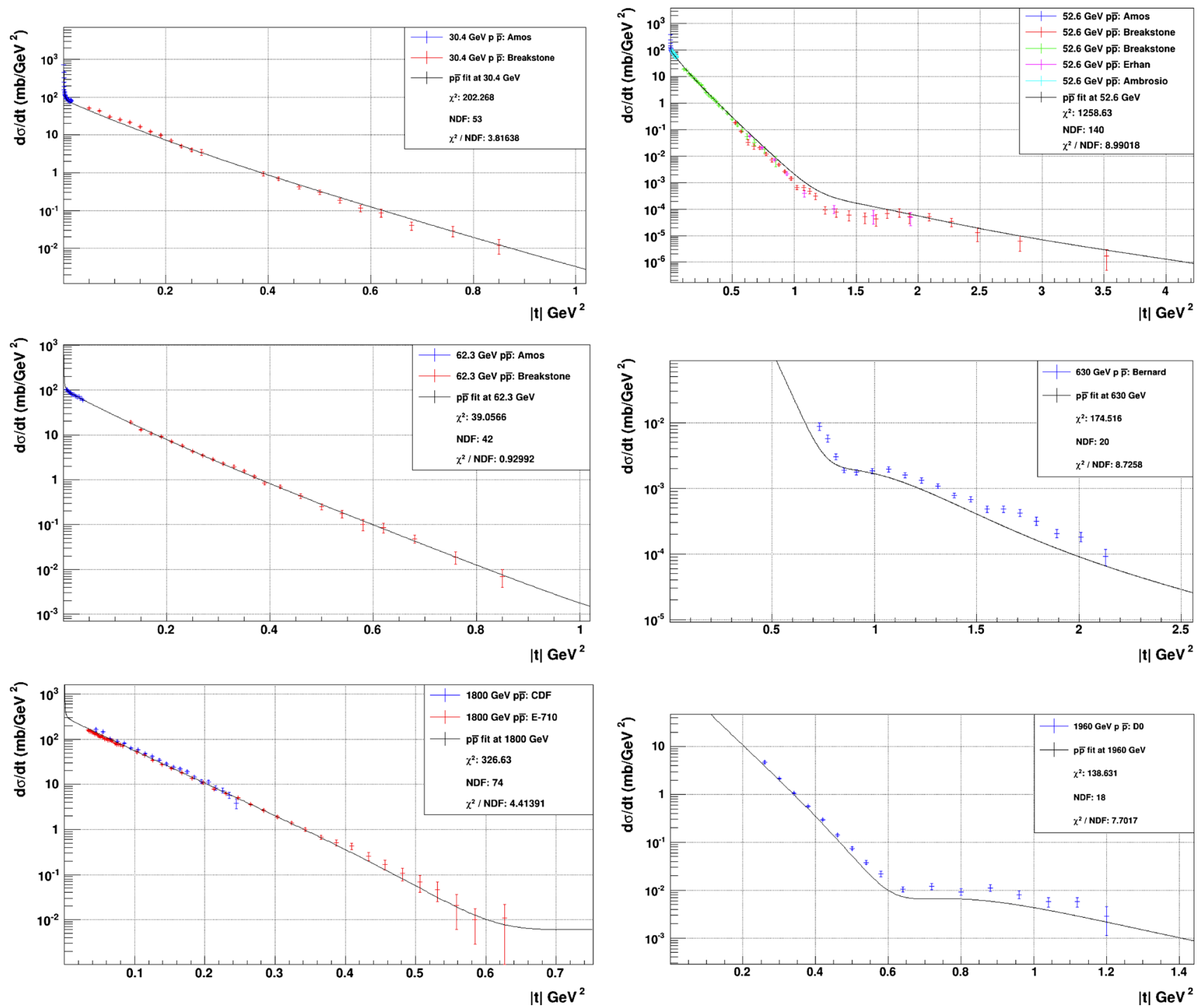

Fig. 24 The $p \bar{p}$ elastic scattering model fit (fitted over all data) shown for a range of $\sqrt{s}$, over the full $t$ range, including the coulomb peak and down to the lower cut on $t$

Table 12 A list of available single diffraction data and its sources

\begin{tabular}{llll}
\hline Experiments & Particles & $\sqrt{s}(\mathrm{GeV})$ & $\begin{array}{l}\text { Diffractive data } \\
\text { sources }\end{array}$ \\
\hline CHLM & $p p$ & $23.4-62.3$ & {$[61,78-80]$} \\
Cool & $p p$ & $13.7-19.4$ & {$[81]$} \\
Akimov & $p p, p d$ & $8.1,12.4,19.3$ & {$[82]$} \\
Schamberger & $p p$ & $16.2-30.7$ & {$[44,83,84]$} \\
UA4 & $p \bar{p}$ & 546 & {$[45,85]$} \\
UA8 & $p \bar{p}$ & 630 & {$[86]$} \\
CDF & $p \bar{p}$ & 546,1800 & {$[87]$} \\
\hline
\end{tabular}



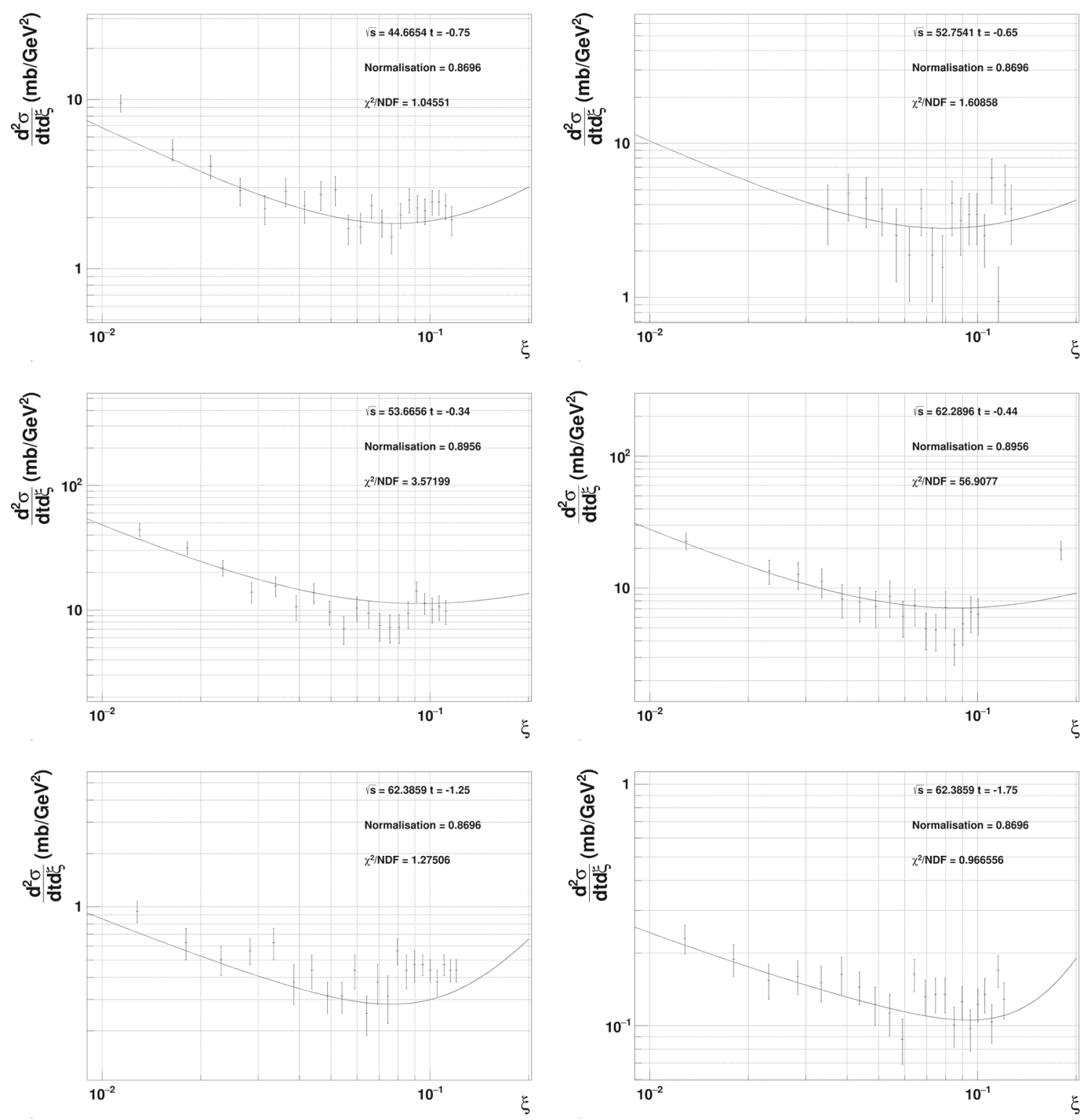

Fig. 25 The SD scattering model fit (fitted over all data) shown for a range of $\sqrt{s}$, over the full $\xi$ range 

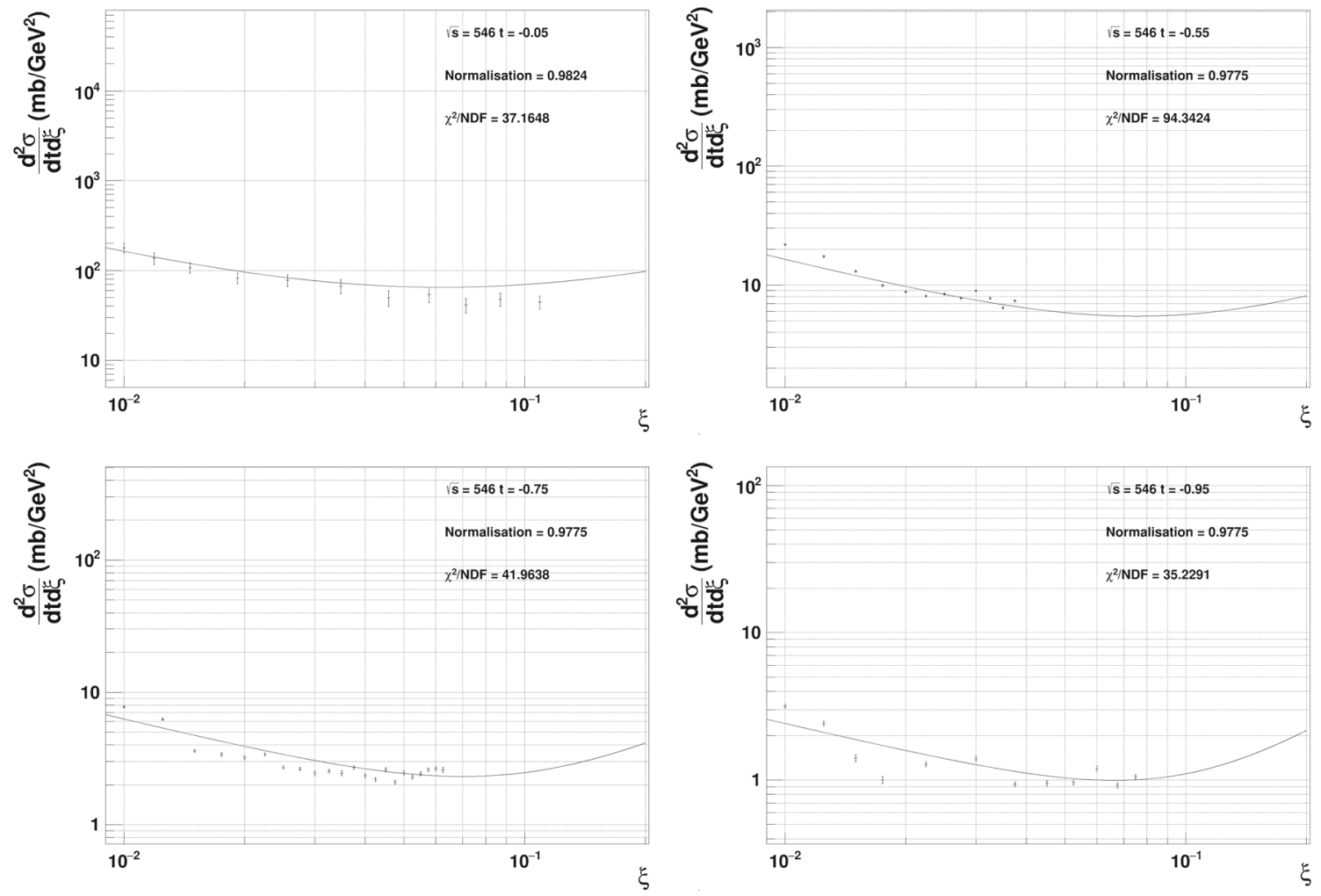

Fig. 26 The SD scattering model fit (fitted over all data) shown for a range of $\sqrt{s}$, over the full $\xi$ range, for $p \bar{p}$ scattering

\section{References}

1. B. Auchmann, T. Baer, M. Bednarek, G. Bellodi, C. Bracco, R. Bruce, F. Cerutti, V. Chetvertkova, B. Dehning, P.P. Granieri, W. Hofle, E.B. Holzer, A. Lechner, E. Nebot Del Busto, A. Priebe, S. Redaelli, B. Salvachua, M. Sapinski, R. Schmidt, N. Shetty, E. Skordis, M. Solfaroli, J. Steckert, D. Valuch, A. Verweij, J. Wenninger, D. Wollmann, M. Zerlauth, Testing beam-induced quench levels of LHC superconducting magnets. Phys. Rev. ST Accel. Beams 18, 061002 (2015)

2. O. Brüning, P. Collier, P. Lebrun, S. Myers, R. Ostojic, J. Poole, P. Proudlock, LHC Design Report (CERN, Geneva, 2004)

3. O. Bruning, L. Rossi, Advanced series on directions in high energy. Physics 24 (2015)

4. R. Bruce, R.W. Assmann, V. Boccone, C. Bracco, M. Brugger, M. Cauchi, F. Cerutti, D. Deboy, A. Ferrari, L. Lari, A. Marsili, A. Mereghetti, D. Mirarchi, E. Quaranta, S. Redaelli, G. RobertDemolaize, A. Rossi, B. Salvachua, E. Skordis, C. Tambasco, G. Valentino, T. Weiler, V. Vlachoudis, D. Wollmann, Phys. Rev. Special Top. Accel. Beams 17, 081004 (2014)

5. R. Bruce, C. Bracco, F. Cerruti, A. Ferrari, A. Lechner, D. Mirarchi, P.G. Ortega, A. Rossi, D.P. Sinuela, V. Vlachoudis, A. Mereghetti, A. Assmann, L. Lari, S.M. Gibson, L.J. Nevay, R.B. Appleby, J. Molson, R. Barlow, A. Toader, H. Rafique, R. Bruce, A. Marsili, S. Redaelli, M. Serluca, B. Salvaucha, C. Tambasco, Integrated simulation tools for collimation cleaning in HL-LHC, in Proceedings of the 5th International Particle Accelerator Conference, MOPRO039, IPAC 2014 (Dresden, Germany, 2014), pp. $160-162$

6. G. Robert-Demolaize, Design and Performance Optimization of the LHC Collimation System. PhD thesis, Université Joseph Fourier, Grenoble (2006)
7. C. Bracco, Commissioning Scenarios and Tests for the LHC Collimation system. $\mathrm{PhD}$ thesis, Universitá degli Studi di Milano, Italy (2008)

8. A. Donnachie, P.V. Landshoff, Nuclear Phys. B 244(2), 322-336 (1984)

9. A. Donnachie, G. Dosch, P.V. Landshoff, O. Nachtmann. Pomeron Physics and QCD (Cambridge University Press, Cambridge, 2002)

10. J. Molson, R.B. Appleby, M. Serluca, A. Toader, R. Barlow. Simulating the LHC collimation system with the accelerator physics library merlin, and loss map results, in Proceedings of the 11th International Computational Accelerator Physics Conference, ICAP 2012, ed. by D. Hecht, M. Marx (Rostock-Warnemünde, Germany, 2012), pp. 12-14

11. M. Serluca, R.B. Appleby, J. Molson, R. Barlow, A. Toader, H. Rafique, Hi-lumi LHC collimation studies with MERLIN code, in Proceedings of the 5th International Particle Accelerator Conference, MOPRI077, IPAC 2014 (Dresden, Germany, 2014), pp. 784-786

12. M. Serluca, R.B. Appleby, J. Molson, R. Barlow, A. Toader, H. Rafique, R. Bruce, A. Marsili, S. Redaelli, B. Salvaucha, C. Tambasco, Comparison of MERLIN/sixtrack for LHC collimation studies, in Proceedings of the 5th International Particle Accelerator Conference, MOPRO046, IPAC 2014 (Dresden, Germany, 2014), pp. $185-187$

13. R.B. Appleby, R.Barlow, J.Molson, H. Rafique, M.Serluca, A.Toader. Merlin source code website. https://github.com/ MERLIN-Collaboration/MERLIN

14. Vladimir Shiltsev, Kip Bishofberger, Vsevolod Kamerdzhiev, Sergei Kozub, Matthew Kufer, Gennady Kuznetsov, Alexander Martinez, Marvin Olson, Howard Pfeffer, Greg Saewert, Vic Scarpine, Andrey Seryi, Nikolai Solyak, Veniamin Sytnik, Mikhail 
Tiunov, Leonid Tkachenko, David Wildman, Daniel Wolff, XiaoLong Zhang, Phys. Rev. ST Accel. Beams 11, 103501 (2008)

15. V.M. Biryukov, V.N. Chepegin, Yu.A. Chesnokov, V. Guidi, W. Scandale, Nuclear Instruments and Methods in Physics Research Section B: Beam Interactions with Materials and Atoms. Relativistic Channeling and Related Coherent Phenomena in Strong Fields, vol. 234(1-2), pp. 23-30 (2005)

16. A. Chao, M. Tigner, Handbook of Accelerator Physics and Engineering (World Scientific, Singapore, 1999)

17. N. Kwak, E. Lohrmann, E. Nagy, M. Regler, W. Schmidt-Parzefall, K.R. Schubert, K. Winter, A. Brandt, H. Dibon, G. Flügge, F. Niebergall, P.E. Schumacher, J.J. Aubert, C. Broll, G. Coignet, J. Favier, L. Massonnet, M. Vivargent, W. Bartl, H. Eichinger, Ch. Gottfried, G. Neuhofer, Phys. Lett. B 58(2), 233-236 (1975)

18. A. Böhm, M. Bozzo, R. Ellis, H. Foeth, M.I. Ferrero, G. Maderni, B. Naroska, C. Rubbia, G. Sette, A. Staude, P. Strolin, G. de Zorzi, Phys. Lett. B 49(5), 491-496 (1974)

19. U. Amaldi, G. Cocconi, A.N. Diddens, Z. Dimčovski, R.W. Dobinson, J. Dorenbosch, P. Duinker, G. Matthiae, A.M. Thorndike, A.M. Wetherell, G. Bellettini, P.L. Braccini, R. Carrara, R. Castaldi, V. Cavasinni, F. Cervelli, T. Del Prete, P. Laurelli, M.M. Massai, M. Morganti, G. Sanguinetti, M. Valdata-Nappi, C. Vannini, A. Baroncelli, C. Bosio, G. Abshire, J. Crouch, G. Finocchiaro, P. Grannis, H. Jöstlein, R. Kephart, D. Lloyd-Owen, R. Thun, Nuclear Phys. B 145(2-3), 367-401 (1978)

20. A. Donnachie, P.V. Landshoff, Phys. Lett. B 123(5), 345-348 (1983)

21. A. Donnachie, P.V. Landshoff, Nuclear Phys. B 267(3-4), 690-701 (1986)

22. A. Donnachie, P.V. Landshoff, Z. Phys. C Part. Fields 2, 55-62 (1979). doi:10.1007/BF01546237

23. A. Donnachie, P.V. Landshoff, Phys. Lett. B 387(3), 637-641 (1996)

24. A. Donnachie, P.V. Landshoff, Elastic scattering at the LHC. arXiv:1112.2485 (2011)

25. J. Molson. Proton scattering and collimation for the $L H C$ and $L H C$ luminosity upgrade. $\mathrm{PhD}$ thesis, University of Manchester Press, Manchester (2014)

26. M.M. Block, R.N. Cahn, Rev. Mod. Phys. 57, 563-598 (1985)

27. R. Cahn, Z. Phys. C Part. Fields 15, 253-260 (1982). doi:10.1007/ BF01475009

28. N. Amos, M.M. Block, G.J. Bobbink, M. Botje, D. Favart, C. Leroy, F. Linde, P. Lipnik, J.-P. Matheys, D. Miller, K. Potter, S. Shukla, C. Vander Velde-Wilquet, S. Zucchelli, Nuclear Phys. B 262(4), 689-714 (1985)

29. R. Brun, F. Rademakers, Root—an object oriented data analysis framework, in AIHENP'96 Workshop, Lausane, vol. 389, pp. 8186 (1996)

30. A. Donnachie, P.V. Landshoff, Phys. Lett. B 727, 500 (2013)

31. A. Donnachie, P.V. Landshoff, Phys. Lett. B 750, 669 (2015)

32. J.V.N. Gribov, Sov. J. Nuclear Phys. 5(5), 138 (1967)

33. A. Donnachie, P.V. Landshoff, Soft diffraction dissociation (2003)

34. E.G.S. Luna, V.A. Khoze, A.D. Martin, M.G. Ryskin, Eur. Phys. J. C 59, 1-12 (2009)

35. A. Donnachie, Unpublished note

36. A.B. Kaidalov, V.A. Khoze, YuF Pirogov, N.L. Ter-Isaakyan, Phys. Lett. B 45(5), 493-496 (1973)

37. S. Ostapchenko, H.J. Drescher, F.M. Liu, T. Pierog, K. Werner, J. Phys. G Nuclear Part. Phys. 28(10), 2597 (2002)

38. E. Gotsman, E. Levin, U. Maor, J.S. Miller, Eur. Phys. J. C 57, 689-709 (2008)

39. M.G. Ryskin, A.D. Martin, V.A. Khoze, Eur. Phys. J. C 54, 199217 (2008)

40. E.G.S. Luna, V.A. Khoze, A.D. Martin, M.G. Ryskin, Eur. Phys. J. C 69(1-2), 95-101 (2010)

41. R.D. Field, G.C. Fox, Nuclear Phys. B 80(3), 367-402 (1974)
42. R.D. Schamberger, J. Lee-Franzini, R. McCarthy, S. Childress, P. Franzini, Phys. Rev. D 17, 1268-1291 (1978)

43. D.R.O. Morrison, Phys. Rev. 165(5), 1699-1702 (1968)

44. R.D. Schamberger, J. Lee-Franzini, R. McCarthy, S. Childress, P. Franzini, Phys. Rev. Lett. 34, 1121-1124 (1975)

45. D. Bernard, M. Bozzo, P.L. Braccini, F. Carbonara, R. Castaldi, F. Cervelli, G. Chiefari, E. Drago, M. Haguenauer, V. Innocente, P. Kluit, B. Koene, S. Lanzano, G. Matthiae, L. Merola, M. Napolitano, V. Palladino, G. Sanguinetti, P. Scampoli, S. Scapellato, G. Sciacca, G. Sette, R. Van Swol, J. Timmermans, C. Vannini, J. Velasco, P.G. Verdini, F. Visco, Phys. Lett. B 186(2), 227-232 (1987)

46. M. Froissart, Phys. Rev. 123(3), 1053-1057 (1961)

47. K. Goulianos, Phys. Lett. B 358(3-4), 379-388 (1995)

48. D.P. Roy, R.G. Roberts, Nuclear Phys. B 77(2), 240-268 (1974)

49. Betty Abelev et al., Eur. Phys. J. C 73, 2456 (2013)

50. S Chatrchyan, Measurement of pseudorapidity distributions of charged particles in proton-proton collisions at $\sqrt{s}=8 \mathrm{TeV}$ by the CMS and TOTEM experiments, in Technical Report. CMS-FSQ12-026. CERN-PH-EP-TOTEM-2014-002. CERN-PH-EP-2014063 (CERN, Geneva, 2014). arXiv:1405.0722 (comments: submitted to the European Physical Journal C)

51. G. Antchev and others (TOTEM Collaboration), Technical Report CERN-PH-EP-2014-260 (CERN, Geneva, 2014)

52. G. Antchev and others (TOTEM Collaboration), EPL (Europhys. Lett.) 101(2), 21003 (2013)

53. V.A. Khoze, A.D. Martin, M.G. Ryskin, Eur. Phys. J. C 73(7) (2013)

54. G. Robert-Demolaize, R. Assmann, S. Redaelli, F. Schmidt, A new version of sixtrack with collimation and aperture interface, in Proceedings of the Particle Accelerator Conference, 2005. PAC 2005, pp. 4084-4086 (2005)

55. R. Bruce et al., Simulations and measurements of cleaning with $100 \mathrm{MJ}$ beams in the LHC, in Proceedings of the 4th International Particle Accelerator Conference (IPAC'13), MOODB202 (IPAC 2013, Shanghai, 2013)

56. http://mad.web.cern.ch $/ \mathrm{mad} /$. Accessed 4 Apr 2016

57. A. Valloni, R.B. Appleby, R. Bruce, A. Mereghetti, J. Molson, $\mathrm{H}$. Rafique et al., Merlin simulations of the LHC collimation system with $6.5 \mathrm{Tev}$ beams, in Proceedings of the 7th International Particle Accelerator Conference (IPAC'16), WEPMW037 (IPAC 2016, Busan, 2016), pp. 2518-2521

58. A. Ferrari, A. Fasso', J. Ranft, P.R. Sala, FLUKA: a multi-particle transport code, in Technical report (2005)

59. S. Agostinelli et al., GEANT4 - a simulation toolkit. Nucl. Instrum. Methods A 506, 250-303 (2003)

60. E. Nagy, R.S. Orr, W. Schmidt-Parzefall, K. Winter, A. Brandt, F.W. Büsser, G. Flügge, F. Niebergall, P.E. Schumacher, H. Eichinger, K.R. Schubert, J.J. Aubert, C. Broll, G. Coignet, H. De Kerret, J. Favier, L. Massonnet, M. Vivargent, W. Bartl, H. Dibon, Ch. Gottfried, G. Neuhofer, M. Regler, Nuclear Phys. B 150, 221-267 (1979)

61. M.G. Albrow, A. Bagchus, D.P. Barber, P. Benz, A. Bogaerts, B. Bosnjaković, J.R. Brooks, C.Y. Chang, A.B. Clegg, F.C. Erné, C.N.P. Gee, P. Kooijman, D.H. Locke, F.K. Loebinger, N.A. McCubbin, P.G. Murphy, D. Radojičič, A. Rudge, J.C. Sens, A.L. Sessoms, J. Singh, D. Stork, J. Timmer, Nuclear Phys. B 108(1), $1-29(1976)$

62. W. Faissler, M. Gettner, J.R. Johnson, T. Kephart, E. Pothier, D. Potter, M. Tautz, S. Conetti, C. Hojvat, D.G. Ryan, K. Shahbazian, D.G. Stairs, J. Trischuk, P. Baranov, J.L. Hartmann, J. Orear, S. Rusakov, J. Vrieslander, Phys. Rev. D 23, 33-42 (1981)

63. A. Breakstone, R. Campanini, H.B. Crawley, G.M. Dallavalle, M.M. Deninno, K. Doroba, D. Drijard, F. Fabbri, A. Firestone, H.G. Fischer, H. Frehse, W. Geist, G. Giacomelli, R. Gokieli, M. Gorbics, P. Hanke, M. Heiden, W. Herr, P.G. Innocenti, E.E. Kluge, J.W. Lamsa, T. Lohse, W.T. Meyer, G. Mornacchi, T. Nakada, M. 
Panter, A. Putzer, K. Rauschnabel, B. Rensch, F. Rimondi, R. Sosnowski, M. Szczekowski, O. Ullaland, D. Wegener, M. Wunsch, Nuclear Phys. B 248(2), 253-260 (1984)

64. U. Amaldi, K.R. Schubert, Nuclear Phys. B 166(2), 301-320 (1980)

65. A. Breakstone, H.B. Crawley, G.M. Dallavalle, K. Doroba, D. Drijard, F. Fabbri, A. Firestone, H.G. Fischer, H. Frehse, W. Geist, G. Giacomelli, R. Gokieli, M. Gorbics, P. Hanke, M. Heiden, W. Herr, E.E. Kluge, J.W. Lamsa, T. Lohse, W.T. Meyer, G. Mornacchi, T. Nakada, M. Panter, A. Putzer, K. Rauschnabel, F. Rimondi, G.P. Siroli, R. Sosnowski, M. Szczekowski, O. Ullaland, D. Wegener, Phys. Rev. Lett. 54, 2180-2183 (1985)

66. M. Ambrosio, G. Anzivino, G. Barbarino, G. Carboni, V. Cavasinni, T. Del Prete, P.D. Grannis, D. Lloyd, M. Owen, G. Morganti, S. Paternoster, S. Patricelli, M. Valdata-Nappi, Phys. Lett. B 115(6), 495-502 (1982)

67. G. Antchev and others (TOTEM Collaboration), EPL (Europhys. Lett.) 95(4), 41001 (2011)

68. G. Antchev and others (TOTEM Collaboration), EPL (Europhys. Lett.) 96(2), 21002 (2011)

69. C. Augier, D. Bernard, J. Bourotte, M. Bozzo, A. Bueno, R. Cases, F. Djama, M. Haguenauer, V. Kundrát, M. Lokajíček, G. Matthiae, A. Morelli, F. Natali, S. Němeček, M. Novák, E. Sanchis, G. Sette, M. Smižanská, J. Velasco, Phys. Lett. B 316(2-3), 448-454 (1993)

70. R. Battiston, M. Bozzo, P.L. Braccini, F. Carbonara, R. Carrara, R. Castaldi, F. Cervelli, G. Chiefari, E. Drago, M. Haguenauer, B. Koene, G. Matthiae, L. Merola, M. Napolitano, V. Palladino, G. Sanguinetti, G. Sciacca, G. Sette, R. van Swol, J. Timmermans, C. Vannini, J. Velasco, F. Visco, Phys. Lett. B 127(6), 472-475 (1983)

71. D. Bernard, M. Bozzo, P.L. Braccini, F. Carbonara, R. Castaldi, F. Cervelli, G. Chiefari, E. Drago, M. Haguenauer, V. Innocente, P. Kluit, S. Lanzano, G. Matthiae, L. Merola, M. Napolitano, V. Palladino, G. Sanguinetti, P. Scampoli, S. Scapellato, G. Sciacca, G. Sette, J. Timmermans, C. Vannini, J. Velasco, P.G. Verdini, F. Visco, Phys. Lett. B 198(4), 583-589 (1987)

72. M. Bozzo, P.L. Braccini, F. Carbonara, R. Castaldi, F. Cervelli, G. Chiefari, E. Drago, M. Haguenauer, V. Innocente, B. Koene, S. Lanzano, G. Matthiae, L. Merola, M. Napolitano, V. Palladino, G. Sanguinetti, S. Scapellato, G. Sciacca, G. Sette, R. van Swol, J. Timmermans, C. Vannini, J. Velasco, P.G. Verdini, F. Visco, Phys. Lett. B 147(4-5), 385-391 (1984)

73. M. Bozzo, P.L. Braccini, F. Carbonara, R. Castaldi, F. Cervelli, G. Chiefari, E. Drago, M. Haguenauer, V. Innocente, B. Koene, S. Lanzano, G. Matthiae, L. Merola, M. Napolitano, V. Palladino, G. Sanguinetti, S. Scapellato, G. Sciacca, G. Sette, R. Van Swol, J. Timmermans, C. Vannini, J. Velasco, P.G. Verdini, F. Visco, Phys. Lett. B 155(3), 197-202 (1985)

74. F. Abe and others (CDF collaboration), Phys. Rev. D 50, 55185534 (1994)

75. D. Bernard, M. Bozzo, P.L. Braccini, F. Carbonara, R. Castaldi, F. Cervelli, G. Chiefari, E. Drago, M. Haguenauer, V. Innocente, P. Kluit, B. Koene, S. Lanzano, G. Matthiae, L. Merola, M. Napolitano, V. Palladino, G. Sanguinetti, P. Scampoli, S. Scapellato, G. Sciacca, G. Sette, R. van Swol, J. Timmermans, C. Vannini, J. Velasco, P.G. Verdini, F. Visco, Phys. Lett. B 171(1), 142-144 (1986)
76. N.A. Amos, C. Avila, W.F. Baker, M. Bertani, M.M. Block, D.A. Dimitroyannis, D.P. Eartly, R.W. Ellsworth, G. Giacomelli, B. Gomez, J.A. Goodman, C.M. Guss, A.J. Lennox, M.R. Mondardini, J.P. Negret, J. Orear, S.M. Pruss, R. Rubinstein, S. Sadr, S. Shukla, I. Veronesi, S. Zucchelli, Phys. Lett. B 247(1), 127-130 (1990)

77. D0 Collaboration, Measurement of the differential cross section $\mathrm{d} \sigma \mathrm{TeV}$, in DO Note 6056-CONF (2010)

78. M.G. Albrow et al., Missing mass spectra in pp inelastic scattering at total energies of $23 \mathrm{GeV}$ and $31 \mathrm{GeV}$. Nuclear Phys. B 72(3), 376-392 (1974)

79. J.C.M. Armitage, P. Benz, G.J. Bobbink, F.C. Erne, P. Kooijman, F.K. Loebinger, A.A. Macbeth, H.E. Montgomery, P.G. Murphy, A. Rudge, J.C. Sens, D. Stork, J. Timmer, Nuclear Phys. B 194(3), 365-372 (1982)

80. P.M. Kooijman, Investigation of Diffraction Dissociation in Proton-Proton Collisions at High Energies. PhD thesis, University of Utrecht (1979)

81. R.L. Cool et al., Diffraction dissociation of $\pi^{ \pm}, K^{ \pm}$, and $p^{ \pm}$at 100 and $200 \mathrm{GeV} / \mathrm{c}$. Phys. Rev. Lett. 47, 701-704 (1981)

82. Y. Akimov, V. Bartenev, R. Cool, K. Goulianos, D.A. Gross, E. Jenkins, E. Malamud, P. Markov, S. Mukhin, D. Nitz, S.L. Olsen, A. Sandacz, S.L. Segler, H. Sticker, R. Yamada, Phys. Rev. Lett. 39, 1432-1435 (1977)

83. S. Childress, P. Franzini, J. Lee-Franzini, R. McCarthy, R.D. Schamberger, Small-momentum-transfer $p-p$ inelastic scattering at 300 gev/c. Phys. Rev. Lett. 32, 389-392 (1974)

84. R.D. Schamberger, J. Lee-Franzini, R. McCarthy, S. Childress, P. Franzini, Mass spectrum of proton-proton inelastic interactions from 55 to $400 \mathrm{gev} / \mathrm{c}$ at small momentum transfer. Phys. Rev. D 17, 1268-1291 (1978)

85. M. Bozzo, P.L. Braccini, F. Carbonara, R. Carrara, R. Castaldi, F. Cervelli, G. Chiefari, E. Drago, M. Haguenauer, B. Koene, G. Matthiae, L. Merola, M. Napolitano, V. Palladino, G. Sanguinetti, G. Sciacca, G. Sette, R. van Swol, J. Timmermans, C. Vannini, J. Velasco, F. Visco, Phys. Lett. B 136(3), 217-220 (1984)

86. A. Brandt et al., Measurements of single diffraction at $\sqrt{s}=630$ gev; evidence for a non-linear $\alpha(t)$ of the pomeron. Nuclear Phys. B 514(1-2), 3-44 (1998)

87. K. Goulianos, J. Montanha, Factorization and scaling in hadronic diffraction. Phys. Rev. D 59, 114017 (1999)

88. G. Antchev and others (The TOTEM Collaboration), Luminosityindependent measurements of total, elastic and inelastic crosssections at $\sqrt{s}=7 \mathrm{TeV}$. EPL 101(2), 21004 (2013)

89. G. Antchev and others (the TOTEM collaboration), Evidence for non-exponential elastic proton-proton differential cross-section at low $|t|=8 \mathrm{TeV}$ by TOTEM. Nuclear Phys. B 899, 527- 546 (2015)

90. G. Antchev and others (the TOTEM collaboration), Measurement of elastic pp scattering at $\sqrt{s}$ parameter and the total cross-section, in Technical Report CERN-PH-EP-2015-325 (CERN, Geneva, 2015)

91. G. Aad and others (the ATLAS collaboration), Measurement of the inelastic proton-proton cross-section at $\sqrt{s}=7 \mathrm{TeV}$ with the ATLAS detector. Nat Commun.2(463), 09 (2011) 